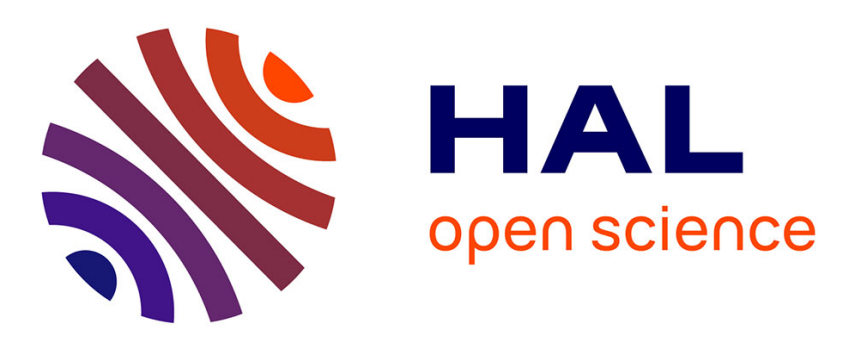

\title{
Bending Carbon Nanoforms for Supramolecular Recognition: A Topological Study on Hemifullerene-Based Aggregates
}

Joaquín Calbo, Juan Aragó, Roberto A. Boto, José Sánchez-Marín, Enrique Ortí, Julia Contreras-García

\section{To cite this version:}

Joaquín Calbo, Juan Aragó, Roberto A. Boto, José Sánchez-Marín, Enrique Ortí, et al.. Bending Carbon Nanoforms for Supramolecular Recognition: A Topological Study on Hemifullerene-Based Aggregates. Journal of Physical Chemistry A, 2018, 122 (4), pp.1124-1137. 10.1021/acs.jpca.7b11746 . hal-02404898

\section{HAL Id: hal-02404898 \\ https: / hal.sorbonne-universite.fr/hal-02404898}

Submitted on 11 Dec 2019

HAL is a multi-disciplinary open access archive for the deposit and dissemination of scientific research documents, whether they are published or not. The documents may come from teaching and research institutions in France or abroad, or from public or private research centers.
L'archive ouverte pluridisciplinaire HAL, est destinée au dépôt et à la diffusion de documents scientifiques de niveau recherche, publiés ou non, émanant des établissements d'enseignement et de recherche français ou étrangers, des laboratoires publics ou privés. 


\title{
Bending Carbon Nanoforms for Supramolecular Recognition: A Topological Study on Hemifullerene-Based Aggregates
}

\author{
Joaquín Calbo, ${ }^{*, a}$ Juan Aragó, ${ }^{a}$ Roberto A. Boto, ${ }^{b}$ José Sánchez-Marín, ${ }^{a}$ Enrique Ortí, ${ }^{*, a}$ Julia \\ Contreras-García*,c,d, \\ a Instituto de Ciencia Molecular, Universidad de Valencia, 46890 Paterna (Valencia), Spain \\ ${ }^{b}$ CICECO-Aveiro, Institute of Materials, University of Aveiro, 3810-193 Aveiro, Portugal \\ c Sorbonne Universités, UPMC Univ Paris 06, UMR 7616, Laboratoire de Chimie Théorique, F-75005, Paris, France \\ ${ }^{d}$ CNRS, UMR 7616, LCT, F-75005, Paris, France
}

Published as part of The Journal of Physical Chemistry special issue "Manuel Yáñez and Otilia Mo Festschrift"

We dedicate this work to Professors Manuel Yáñez and Otilia Mó, outstanding scientists and wonderful friends, in light of their efforts in promoting the theoretical and computational chemistry in Spain.

\section{ABSTRACT}

Buckybowls have risen as appealing fullerene fragment derivatives. Their intrinsic curvature has been exploited in the generation of host-guest supramolecular assemblies, not only through concave-convex complementarity but also through less-known concave-concave staggered arrangements. Whereas the stabilization of bowl-in-bowl dispositions has been ascribed to efficient $\pi-\pi$ forces together with favorable dipole-dipole interactions, a detailed analysis on the forces guiding the formation of the staggered arrangements is missing so far. Herein, we present a thorough theoretical characterization of bowl-in-bowl vs. staggered hemifullerene-based homodimers and heterodimers with the electron-donor truxTTF molecule, as test cases, under the density functional theory and by means of chemical bonding techniques. Our results clearly reveal strong and localized noncovalent signatures, together with an enhanced orbital interaction, associated with $\mathrm{CH}-\pi$ and sulfur-mediated interactions governing the staggered formation. Bending the fullerene fragment is demonstrated to favor the stabilization in both homo- and heterodimers, in good accord with the depletion in the r-electron density calculated upon increasing the buckybowl curvature. The optimal buckybowl curvature for the highest interaction energy is however dependent on the type of supramolecular assembly (bowl-in-bowl vs. staggered) and the concave region to which hemifullerene approaches truxTTF. Interestingly, two regimes are found as a function of buckybowl curvature for hemifullerene homodimers: bowl-inbowl dispositions are calculated more stable at low curvatures whereas staggered dimers prevail for highly curved buckybowls. Our results highlight the potential of discrete $\mathrm{CH}-\pi$ and S-mediated interactions to generate unconventional staggered supramolecular arrangements towards the development of a new and unexplored host-guest chemistry. 


\section{INTRODUCTION}

Since the discovery of fullerene $\mathrm{C}_{60}$, undoubtedly the most-known curved carbon architecture, bowl-shaped nanostructures have attracted an increasing interest in the scientific community as chemical models to better understand their parent carbon alloform. ${ }^{1}$ Fullerenes are already consolidated in materials science, and have spurred applications as $n$-type semiconducting materials in organic solar cells. ${ }^{2-4}$ In this context, they have been widely studied as electron acceptors in fundamental investigations of photoinduced electron-transfer processes when combined with electron donors in covalent and noncovalent dyads. ${ }^{5-8}$ In contrast, the application of bowl-shaped fullerene fragments has been limited so far, only being exploited in coordination chemistry ${ }^{9}$ and, recently, in the generation of receptors for fullerenes. ${ }^{10-12}$

The bowl shape of carbon nanoforms has been skillfully used to build up concave hosts for fullerene recognition in concave-convex supramolecular assemblies. The most prominent example of these hosts is probably the so-called buckycatcher reported by Sygula et al., ${ }^{13}$ which bears two concave corannulene moieties to form a molecular pincer that strongly binds $C_{60}$ through a large surface of $\pi-\pi$ noncovalent interactions. Thenceforth, the supramolecular host-guest chemistry of carbon nanoforms has experienced an increasing development in the design of new molecular receptors that maximize the concave-convex complementarity. ${ }^{14-15}$

Bowl-shaped carbon nanoforms (or buckybowls) can be easily synthesized in pure form with a well-defined molecular structure. Due to this, an increasingly array of curved molecular fragments, for which x-ray crystallography data are commonly available, have been reported in the last decade. ${ }^{16-20}$ A particularly interesting example of buckybowls is the so-called hemifullerene $\mathrm{C}_{30} \mathrm{H}_{12}$ (Figure 1a), whose carbon structure corresponds to a half of fullerene $\mathrm{C}_{60}{ }^{21}$ For this fullerene fragment, two crystal polymorphs were found, each of which showing a different packing motif, originating from the interaction between the $\mathrm{C}_{30} \mathrm{H}_{12}$ molecules. In the trigonal polymorph, bowl-inbowl columnar stacks were found, an arrangement dominated by $\pi-\pi$ interactions between the concave and convex regions of adjacent molecules along the stack. In the orthorhombic polymorph, each hemifullerene inserts one of its three peripheral six-membered rings into the cavity of a neighboring molecule, forming dimers presumably stabilized by both $\mathrm{CH}-\pi$ and $\pi-\pi$ noncovalent interactions. The exploitation of these $\mathrm{CH}-\pi$ forces in supramolecular buckybowl arrangements might become an interesting approach for developing a new host-guest chemistry.

Despite the great success in the synthesis of size- and curvature-dependent buckybowls, only few theoretical works have been devoted to categorize the role that the curved shape in these carbon nanoforms plays for increasing the efficiency in the supramolecular recognition of other organic structures, as well as to understand the nature of the noncovalent forces that stabilize the resulting assembly. In the seminal work of Sygula et al., ${ }^{22}$ theoretical calculations at the SCS-MP2 level on different corannulene dimers (native, planar or " $\mathrm{C}_{60}$-like") demonstrated that the curvature of the conjugated carbon network does not disable their ability to form $\pi-\pi$ stacked assemblies. In contrast to the planar systems, part of the stabilization in these bowl-shaped dimers was attributed to attractive electrostatic dipole-dipole contributions.

Recent theoretical work of E. M. Cabaleiro-Lago et al. ${ }^{23}$ on buckybowls with different size and structure has demonstrated that the shape of the buckybowl plays a crucial role in its "catching" ability, being the addition of flaps at the bowl rim by benzannelation an effective strategy for 
enhancing the interaction with fullerenes. Likewise, the introduction of methyl groups in the periphery of corannulene and sumanene was demonstrated to enhance the supramolecular interaction through effective $\mathrm{CH}-\pi$ forces. ${ }^{24}$ The effects of buckybowl curvature on $\pi-\pi$ interactions were also theoretically examined by D. Sherrill et al., ${ }^{25}$ through modelling of coronene and corannulene derivatives with variable degree of curvature. In this work, bending was demonstrated to redistribute the electronic charge in the $\pi$-cloud and create a net dipole moment in the $\pi$-system, leading to enhanced intermolecular electrostatic interactions in the concaveconvex geometries. Curvature of both monomers was additionally shown to have a geometric effect on the interaction by decreasing the average $\mathrm{C}-\mathrm{C}$ distance between monomers, and by increasing the magnitude of both favorable London dispersion interactions and unfavorable exchange-repulsion interactions.

Notwithstanding the great theoretical effort made to understand the noncovalent interactions giving rise to stacked $\pi-\pi$ supramolecular complexes in curved carbon nanoforms, the study of staggered arrangements, such as those found for the orthorhombic polymorph of hemifullerene, has been neglected so far. Recently, some of us have reported the first donor-acceptor supramolecular assembly of a fullerene fragment mimicking the charge-transfer behavior of $\mathrm{C}_{60}$, in which the hemifullerene buckybowl interacts with the donor truxTTF moiety (Figure 1a) through either bowl-in-bowl or staggered arrangements. ${ }^{26}$ Interestingly, high-level DLPNO-CCSD(T) theoretical calculations demonstrated that the staggered disposition, in which the donor and the acceptor interact through a combination of $\pi-\pi, \mathrm{CH}-\pi$ and $n-\pi$ contacts, is the most stable dimer, ${ }^{27}$ in good accord with experimental NMR evidences. ${ }^{28}$

To provide deep insight into the origin and nature of the noncovalent forces governing the selfassembly of unconventional staggered concave-concave arrangements involving buckybowls, we analyze herein the supramolecular assembly of hemifullerene $\mathrm{C}_{30} \mathrm{H}_{12}$, either in a homodimer species or in a heterodimer with the electron donor truxTTF host, through chemical bonding techniques. The intermolecular region of bowl-in-bowl vs. staggered dimers has been examined by scrutiny of the electron density, whereas the $\pi$-stacking ability of hemifullerene upon bending has been assessed using the localized orbital locator descriptor. The effect of molecular curvature in the bowl-in-bowl vs. staggered arrangements is disentangled for both homo- and heterodimers, and optimal buckybowl bendings are found for each regime. Our results highlight the role of discrete $\mathrm{CH}-\pi$ and sulfur-mediated forces in the stabilization of staggered assemblies for the development of a new host-guest chemistry that exploits other than the ubiquitous concave-convex complementarity.

\section{COMPUTATIONAL DETAILS}

The noncovalent interaction $(\mathrm{NCl})$ index (also known as the reduced density gradient) allows to analyze the noncovalent forces governing the different supramolecular assemblies and was calculated using the NCIPLOT-3.0 program. ${ }^{29-30}$ The $\mathrm{NCl}$ method is based on the relationship between the reduced density gradient $(s)$ and the electron density $(\rho)$, according to:

$$
s=\frac{1}{2\left(3 \pi^{2}\right)^{1 / 2}} \frac{|\nabla \rho|}{\rho^{4 / 3}}
$$


When a weak inter- or intramolecular interaction is present, there is a significant change in the density gradient between the interacting atoms. Roots of $s$ are associated with electron density critical points, which can be assigned to attractive or repulsive interactions by examining the second eigenvalue $\left(\lambda_{2}\right)$ of the electron-density Hessian matrix $\left(\nabla^{2} \rho=\lambda_{1}+\lambda_{2}+\lambda_{3}\right)$. The reduced density gradient $s$ can be represented in a 2D plot against the density, including the sign of $\lambda_{2}$ $\left(\operatorname{sign}\left(\lambda_{2}\right) \rho\right)$ and thus yielding a semi-quantitative picture of the intensity and nature of the interaction (negative $\lambda_{2}$ values for attractive and positive $\lambda_{2}$ values for repulsive forces).

In the 3D graphical representation of the reduced density gradient, a 3D grid-point space is obtained for $s$ values lower than a threshold and within a range of $\operatorname{sign}\left(\lambda_{2}\right) \rho$. Green regions refer to weak dispersion-based interactions (small values of $\operatorname{sign}\left(\lambda_{2}\right) \rho$, either positive or negative), whereas bluish contours indicate stronger interactions (large negative $\operatorname{sign}\left(\lambda_{2}\right) \rho$ values). Reddish $\mathrm{NCl}$ surfaces are indicative of repulsive/steric noncovalent interactions (large positive $\operatorname{sign}\left(\lambda_{2}\right) \rho$ values).

The molecular electron density computed from the sum of atomic contributions, the so-called promolecular density, was employed as an approach to $\rho$. The $\rho$ and $s$ cutoffs were kept as default ( 0.20 and 1.00 au, respectively). Sign $\left(\lambda_{2}\right) \rho$ values from -0.02 to +0.02 and an isovalue of $s=0.3$ were set in the 3D plots to recover all the noncovalent interactions in the system. The INTERMOLECULAR option was set to 0.95 to turn off all the interactions where at least $95 \%$ of the density comes from a single molecule.

In order to quantify the amount of noncovalent interactions through the $\mathrm{NCl} 3 \mathrm{D}$ plots, we have also calculated the electron density within the density functional theory (DFT) approach at the PBE0-D3/6-31G** level of theory. On the one hand, we have analysed the electron density at the bond critical points (bcps) following the Bader's approach according to the Quantum Theory for Atoms in Molecules (QTAIM) ${ }^{31}$ using the AIMAll suite of programs. ${ }^{32}$ Only bcps with electron density $<0.016$ au were considered. For delocalized interactions, which are crucial in the carbonbased systems, $\mathrm{NCl}$ volumes were also calculated. ${ }^{33}$ Integration of volumes within the $\mathrm{NCI}$ regions $\left(\mathrm{V}_{\mathrm{T}}\right)$ was attained by means of a modified version of NCIPLOT-3.0 $0^{29-30}$ using the SPLINE technique. This quantity is directly related to the amount of noncovalent interactions (both attractive and repulsive) present in the system.

The planar hemifullerene structure was calculated as a transition state characterized by an imaginary frequency of $125.18 \mathrm{~cm}^{-1}$ at the PBE0-D3/6-31G** level. This structure together with the imaginary frequency connecting the two mirror-image curved conformations were employed to generate a series of hemifullerene geometries with variable curvature using the intrinsic reaction path (IRC) technique as implemented in Gaussian 09.D01. ${ }^{34}$ The IRC structural path was also calculated at the PBE0-D3/6-31G** level, using a step size of 0.50 Bohr and with symmetry constraints disabled (NoSymm). The intramolecular geometry parameters (distances, angles and dihedrals) obtained from the IRC structures were frozen in the subsequent analyses.

The LOLIPOP index ${ }^{35}$ was calculated to measure the $\pi$-density depletion of the hemifullerene derivative as a function of its curvature. The LOLIPOP index is based on the Localized Orbital Locator $(\mathrm{LOL})^{36}$, a function of the kinetic energy density which offers an intuitive depiction of $\pi-$ bonds and detailed information on the nature and location of electron pairs. The LOL function is calculated as 


$$
\mathrm{LOL}=\frac{1}{1+\frac{\tau}{\tau_{\mathrm{TF}}}}
$$

where

$$
\tau=\frac{1}{2} \sum_{i}\left|\nabla \phi_{i}\right|^{2}
$$

is the Kohn-Sham kinetic energy density of the system expressed in terms of spin orbitals $\phi_{i}$, and

$$
\tau_{\mathrm{TF}}=\frac{3}{5}\left(6 \pi^{2}\right)^{2 / 3} \rho^{5 / 3}
$$

is the corresponding Thomas-Fermi kinetic energy density in the uniform electron gas. Therefore, LOL $>0.5$ reveals electrons locally "slower" (less kinetic energy density) than in the uniform electron gas, and LOL $<0.5$ electrons locally "faster" than in the uniform electron gas.

The LOL-based index LOLIPOP (LOL Integrated Pi Over Plane) corresponds to the integral of LOL values $>0.55$ computed from the $\pi$-density $\left(L L_{\pi}\right)$ over a $1.94 \AA$ radius cylinder centered in the middle of the target ring. The chosen integration radius of $1.94 \AA$ corresponds to the average between the $\mathrm{C}$ and $\mathrm{H}$ radii in benzene. The use of other radii within a small range has been demonstrated not to alter the relative trends. ${ }^{35}$ The $\mathrm{LOL}_{\pi}$ surface was calculated by means of the DGRID-4.6 software ${ }^{37}$ using all $\pi$-type orbitals with a grid mesh of 0.05 Bohr, and the LOLIPOP index was obtained through the LOLIPOP script. ${ }^{35}$

It is interesting to note that both topological indexes used here, NCl and LOLIPOP, are able to reveal chemical bonding by virtue of their connection to the kinetic energy density. Whereas the $\mathrm{NCl}$ kernel $(s)$ is related to the scaled bosonic kinetic energy density, $\tau_{w}$, through the definition of the dimensionless variable $t_{\text {bose }},{ }^{38}$ the LOL kernel $\left(t_{\mathrm{LOL}}\right)$ is based on the same scaling of the real fermionic kinetic energy density, $\tau$ :

$$
\begin{gathered}
t_{\mathrm{bose}}=\frac{\tau_{w}}{\tau_{\mathrm{TF}}}=\frac{5}{3} s^{2} \\
t_{\mathrm{LOL}}=\frac{\tau}{\tau_{\mathrm{TF}}}
\end{gathered}
$$

where $\tau_{\mathrm{TF}}$ is used as scaling factor. Interestingly, these two quantities are further related through the electron localization function (ELF) kernel, $\chi$, according to:

$$
\chi=\frac{\tau-\tau_{w}}{\tau_{\mathrm{TF}}}=t_{\mathrm{LOL}}-t_{\mathrm{bose}}
$$

Finally, the electron density rearrangement taking place upon supramolecular complex formation was also analyzed by exploiting the properties of the Natural Orbitals for Chemical Valence 
(NOCV). ${ }^{39}$ The NOCV theory is based on the deformation density $\left(\Delta \rho_{\text {orb }}\right)$, or differential density between the supermolecule $(\rho)$ and the sum of the densities of the constituting $N$ fragments $\left(\rho^{0}\right)$, according to:

$$
\Delta \rho_{\text {orb }}=\rho-\rho^{0}=\rho-\sum_{i}^{N} \rho_{i}
$$

Thus, the orbital interaction energy ( $\left.\Delta E_{\text {orb }}\right)$ is given by:

$$
\Delta E_{\text {orb }}=E[\rho]-E\left[\rho^{0}\right]
$$

where $E[\rho]$ is the energy of the final supermolecule, and $E\left[\rho^{0}\right]$ is the energy associated with the normalized and antisymmetrized wave function obtained from the product wave function of the constituting fragments.

The NOCV eigenvalues and the corresponding energy contributions were calculated using the Extended Transition State (ETS) method of Ziegler. ${ }^{39}$ The orbital interaction term $\left(\Delta E_{\text {or }}\right)$ was obtained according to the NOCV/ETS decomposition scheme as implemented in ORCA 4.0. ${ }^{40} \mathrm{~A}$ very fine integration grid (grid5) and thresholds (verytightscf) were used, along with the hybrid PBEO functional and the 6-31G** basis set previously chosen in our SCF calculations.

\section{RESULTS AND DISCUSSION}

\subsection{Electron Density Analysis}

The hemifullerene $\mathrm{C}_{30} \mathrm{H}_{12}$ (Figure 1a) crystallizes in two polymorphs, i.e. trigonal and orthorhombic phases, from which two types of homodimers can be extracted: bowl-in-bowl and staggered arrangements, respectively. Both dispositions were optimized at the PBE0-D3/6-31G** level of theory and the minimum-energy structures of the bowl-in-bowl (1) and staggered (2) dimers are shown in Figure $1 b$. 
a)
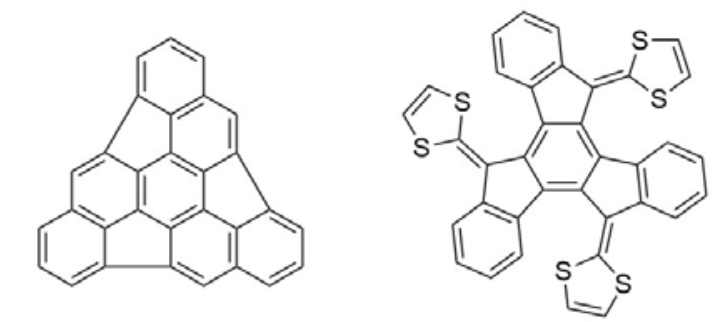

Hemifullerene $-\mathrm{C}_{30} \mathrm{H}_{12}$

truxTTF

b)

1

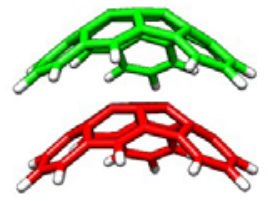

2

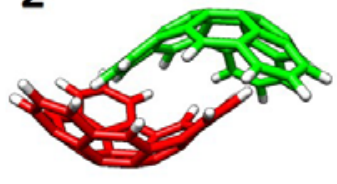

\section{3}
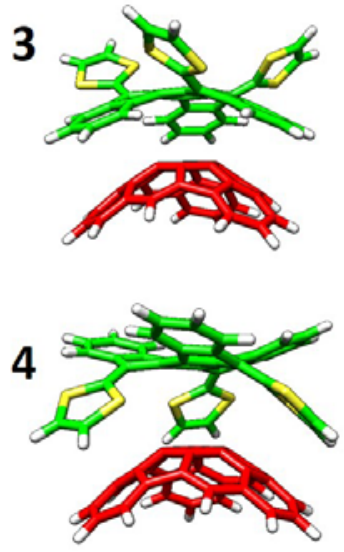
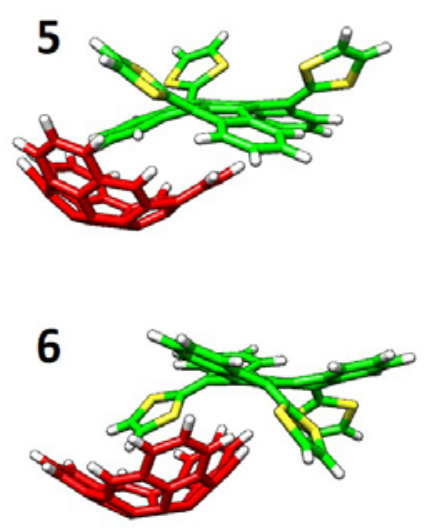

Figure 1. a) Chemical structure of hemifullerene $\mathrm{C}_{30} \mathrm{H}_{12}$ and truxTTF compounds. b) Minimumenergy structures calculated for the different bowl-in-bowl and staggered arrangements of hemifullerene homodimers ( $\mathbf{1}$ and $\mathbf{2}$, top) and truxTTF-hemifullerene heterodimers (3-6, bottom).

Recently, some of us reported the first donor-acceptor supramolecular complex of a fullerene fragment that mimics the photoinduced charge-transfer behavior of related donor-acceptor fullerene aggregates. ${ }^{26}$ In this complex, the hemifullerene is coupled to a tetrathiafulvalene (TTF)based derivative of the truxene core decorated with three dithiole rings (the so-called truxTTF). The truxTTF-hemifullerene associate may be arranged in several spatial dispositions, similarly to the arrangements found in the two polymorphs of hemifullerene. Figure $1 \mathrm{~b}$ summarizes the minimum-energy structures calculated for the four most stable dimeric arrangements (3-6) found in the truxTTF-hemifullerene heterodimer. In structures $\mathbf{3}$ and $\mathbf{4}$, the convex surface of the $\mathrm{C}_{30} \mathrm{H}_{12}$ bowl perfectly matches the two concave cavities of the truxTTF host; that is, either through the cavity formed by the carbon backbone (3) or through the cavity formed by the central benzene ring and the three dithiol rings (4). Structures $\mathbf{3}$ and $\mathbf{4}$ can therefore be seen as bowl-in-bowl arrangements, in analogy with $\mathrm{C}_{30} \mathrm{H}_{12}$ homodimer 1 . Otherwise, the concave cavities of truxTTF and $\mathrm{C}_{30} \mathrm{H}_{12}$ can also interact, giving rise to heterodimers in which either a benzene or a dithiol ring of the truxTTF moiety is placed inside the concave region of the hemifullerene bowl (structures 5 
and 6, respectively). These arrangements are better described as staggered dispositions, in analogy to that extracted from the orthorhombic crystal of hemifullerene (2). The optimized heterodimeric structures 3-6 were reported within a narrow interaction energy range from -20 to $-30 \mathrm{kcal} / \mathrm{mol}$ at the revPBEO-D3/cc-pVTZ level. ${ }^{26}$ Structure 6 was demonstrated to be the most stable arrangement with a binding energy of $-28.5 \mathrm{kcal} / \mathrm{mol}$. The energetic order predicted at the DFT level for structures 3-6 was confirmed by high-level DLPNO-CCSD(T) theoretical calculations. ${ }^{27}$

In order to understand the origin of the forces driving the self-assembly between two hemifullerenes, and between hemifullerene and the donor truxTTF moiety, we analyzed the reduced density gradient ( $\mathrm{NCl}$ index) using the NCIPLOT software. Figure 2a displays the 3D plots of $\mathrm{NCl}$ surface for the two most stable homodimers of benzene (parallel displaced or PD, and Tshape or T) and hemifullerene (bowl-in-bowl 1 and staggered 2). Whereas the PD benzene dimer provides an extended surface of $\mathrm{NCl}$ with close-to-zero density values (green) at $s=0.3$ (i.e. critical points have a low persistence), the $\mathrm{T}$ benzene dimer, although having a much smaller $\mathrm{NCl}$ surface, shows stronger localized interactions evidenced by blue regions. The bowl-in-bowl hemifullerene dimer 1 provides a large $\mathrm{NCl}$ surface with greenish coloring corresponding to densities close to 0 , as a similar situation to the parallel benzene dimer. Interestingly, a donut hole is predicted in the central region of 1 (Figure 1a), suggesting that the main stabilization arises from the intermolecular contacts at the periphery of the dimer. Otherwise, the staggered hemifullerene dimer $\mathbf{2}$ shows a more discontinuous $\mathrm{NCl}$ surface with a large extended green surface, and several gulfs and bays with blue/red regions, indicating a complex scenario of noncovalent interactions.

a)

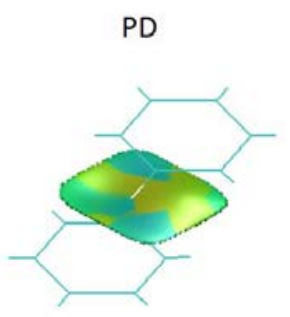

b)

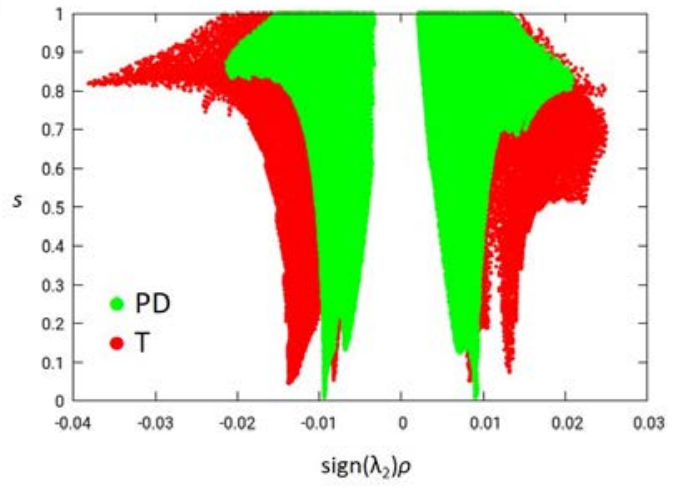

T

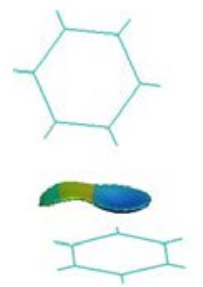

1

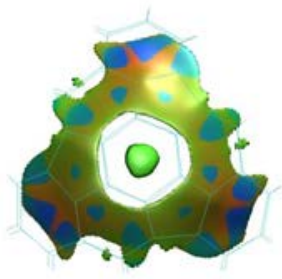

2

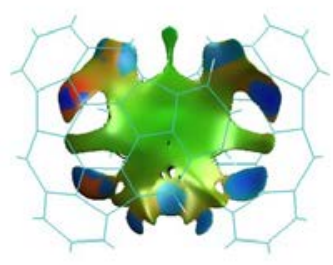

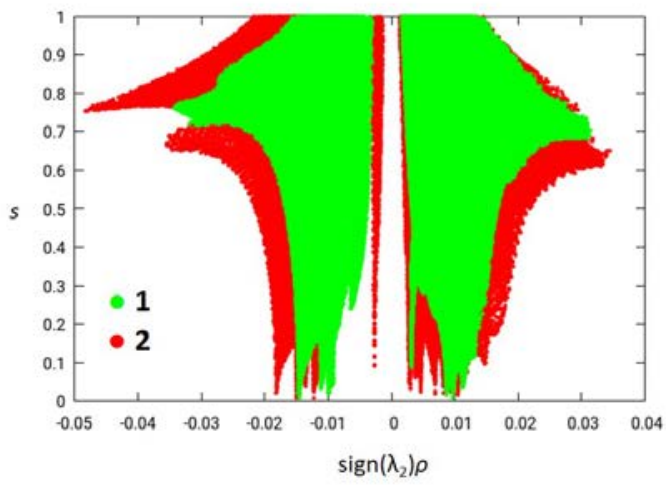

Figure 2. a) Intermolecular $\mathrm{NCl}$ surfaces calculated for benzene and hemifullerene homodimers with an $s$ isovalue of 0.3 and a $\operatorname{sign}\left(\lambda_{2}\right) \rho$ range of -0.02 to +0.02 . b) Reduced density gradient 2D plots for benzene (left) and hemifullerene (right) homodimers. Benzene dimer structures were extracted from the $\mathbf{S 6 6}$ database. ${ }^{41}$ 
To further categorize the nature of the interacting forces governing our assemblies, we analyzed the reduced density gradient by plotting $s$ vs. $\operatorname{sign}\left(\lambda_{2}\right) \rho$. As shown in Figure $2 b$, the PD benzene and bowl-in-bowl hemifullerene 1 homodimers are characterized by spikes close to $\rho=0$, indicative of weak noncovalent interactions between the surface of the $\pi$-conjugated moieties. Otherwise, $T$ benzene and hemifullerene $\mathbf{2}$ homodimers are predicted with peaks in $\mathbf{s}$ at larger positive and negative abscissa values, indicative of stronger attractive and repulsive noncovalent interactions. For example, $s$-spikes at $\operatorname{sign}\left(\lambda_{2}\right) \rho=-0.015$ are predicted for $\mathrm{T}$ benzene dimer, and even stronger interactions are represented by peaks in $s$ at $\operatorname{sign}\left(\lambda_{2}\right) \rho \approx-0.020$ for homodimer 2 . As a reference, a typical H-bond interaction in a water dimer is calculated as a peak at $\operatorname{sign}\left(\lambda_{2}\right) \rho \approx-0.025$. PD benzene and hemifullerene 1 homodimers are therefore characterized by smooth and extended surfaces of $\mathrm{NCl}$ contacts typical of $\pi-\pi$ stacking interactions (see $\mathrm{C}-\mathrm{C}$ intermolecular bond critical points in Table S1, especially those at high $\rho$ values). In contrast, T benzene and hemifullerene 2 homodimers are stabilized not only by dispersion delocalized noncovalent forces but especially by discrete, and relatively strong, localized $\mathrm{CH}-\pi$ interactions ( $\mathrm{C}-\mathrm{H}$ intermolecular bond critical points in Table S2). The absence of noticeable polar interactions leads to a very flat $\mathrm{NCl}$ profile for homodimers $\mathbf{1}$ and 2, which is reflected in the high $\mathrm{NCl}$ volume calculated for these two compounds $\left(\mathrm{V}_{\mathrm{T}}\right.$ in Table 1$)$.

Donor-acceptor truxTTF-hemifullerene heterodimers were then examined following the same $\mathrm{NCl}$ analysis (Figure 3). Bowl-in-bowl dimers $\mathbf{3}$ and $\mathbf{4}$ show a large $\mathrm{NCl}$ surface spreading over the closest contacts between the donor and acceptor moieties (Figure 3a). Subtle differences are observed between the two possible orientations in which $\mathrm{C}_{30} \mathrm{H}_{12}$ can interact with truxTTF. In heterodimer 3, the $\mathrm{NCl}$ surface extends practically over the hemifullerene size, with $\lambda_{2}>0$ (repulsive, in red) regions and $\lambda_{2}<0$ (attractive, in blue) spots in the central intermolecular part. In heterodimer 4, a typical green benzene...benzene $\mathrm{NCl}$ surface is predicted in the central region, which extends across the interaction between hemifullerene and the dithiol rings. The $\mathrm{NCl}$ patterns of $\mathbf{3}$ and $\mathbf{4}$ thus differ from each other and from that obtained in homodimer $\mathbf{1}$. These results highlight differences in the supramolecular interaction owing to different concave-convex complementarities, and to the presence of heteroatoms. On the other hand, the $\mathrm{NCl}$ profiles for $\mathbf{5}$ and 6 (Figure 3a) are found with a similar complexity as that obtained for the staggered homodimer 4 (Figure 2a). Minimum-energy geometries for 5 and $\mathbf{6}$ provide not only extended $\mathrm{NCl}$ surfaces in green but also sharp bays associated with strong attractive interacting regions (surfaces in blue), indicative of effective discrete forces, and with a significant participation of the dithiol rings. 
a)

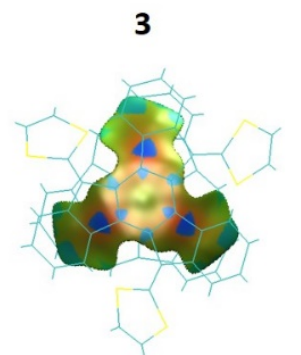

b)
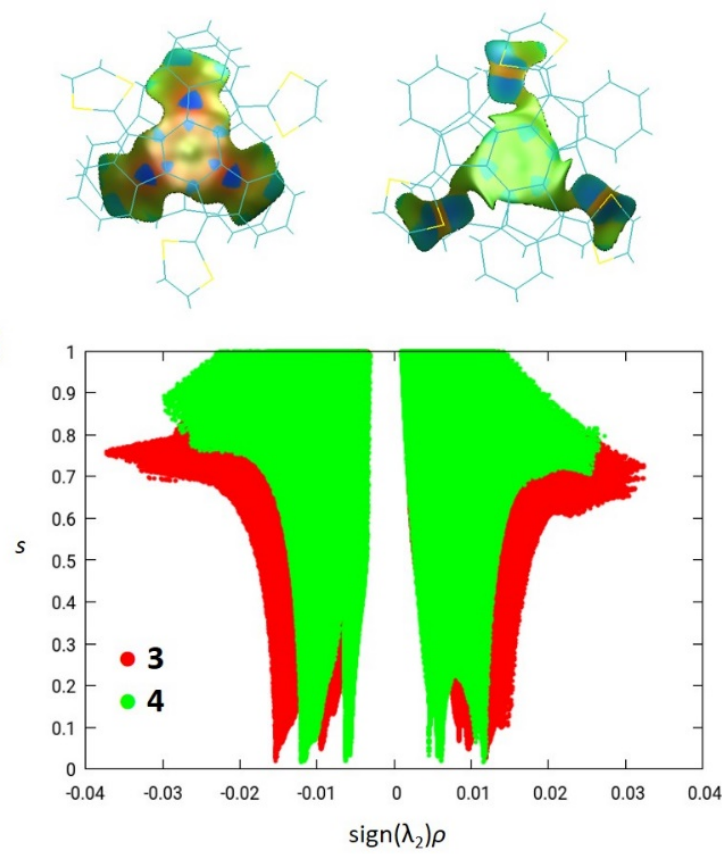
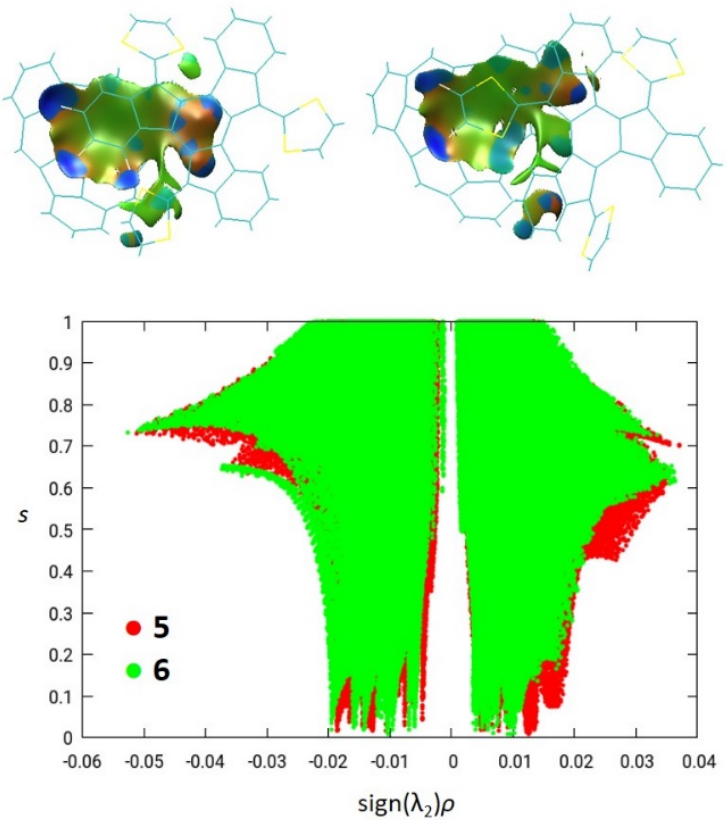

Figure 3. a) Intermolecular $\mathrm{NCl}$ surfaces calculated for truxTTF-hemifullerene heterodimers with an $s$ isovalue of 0.3 and a $\operatorname{sign}\left(\lambda_{2}\right) \rho$ range of -0.02 to +0.02 . b) Reduced density gradient $(s)$ diagrams for truxTTF-hemifullerene heterodimers.

The analysis of the reduced density gradient 2D plots gives further insight into the nature of the weak interactions governing our heterodimeric assemblies. Despite geometrical similarities in which bowl-in-bowl heterodimers $\mathbf{3}$ and $\mathbf{4}$ arrange in the tridimensional space, strong differences are computed in the 2D s-plots. As observed in Figure $3 \mathrm{~b}$, dimer 3 provides s-spikes at large $\operatorname{sign}\left(\lambda_{2}\right) \rho$ values, both positive and negative, compared to dimer 4 . This suggests, as already captured by the colored $\mathrm{NCl}$ surfaces in Figure 3a, strong $\mathrm{C}-\mathrm{C}$ interactions (Table S3) in dimer 3, mainly localized in the central carbon core-core proximities (planar region). Conversely, dimer 4 provides $s$-peaks closer to $\operatorname{sign}\left(\lambda_{2}\right) \rho=0$, indicative of softer noncovalent interactions, with the strongest attractive/repulsive forces associated to the sulfur (truxTTF) - carbon (hemifullerene) contacts (Figure 3 and Table S4). Moving to the staggered heterodimers 5 and 6, s-spikes at higher electron densities are obtained. These are related to the direct $\mathrm{CH}-\pi, \mathrm{S}-\pi$ and $\mathrm{S}-\mathrm{H}$ interactions (Table S5-S6 and Figure S3). A very similar distribution of $s=0$ peaks is obtained for both dimers, with a density range spreading from nearly $\operatorname{sign}\left(\lambda_{2}\right) \rho=0$ to \pm 0.02 . However, in-depth analysis of the bond critical points indicates that relatively strong $S-\pi$ interactions are only present in the case of 6 (Figure S3). The electron densities in the heterodimers are locally contracted due to the presence of sulfur atoms (Figure S3), leading to smaller integrated $\mathrm{NCl}$ volumes than in the homodimer species (Table 1).

The electron density rearrangement that takes place upon supramolecular dimer formation was analyzed using the Natural Orbital for Chemical Valence (NOCV) decomposition technique (see 
Computational Details). The orbital interaction energies ( $\left.\Delta E_{\text {orb }}\right)$ are summarized in Table 1. $A$ large orbital interaction of $-462.6 \mathrm{kcal} / \mathrm{mol}$ is computed for the staggered dimer $\mathbf{2}$, whereas a smaller $\Delta E_{\text {orb }}$ value of $-189.8 \mathrm{kcal} / \mathrm{mol}$ is obtained for bowl-in-bowl dimer 1. Likewise, moving to donoracceptor heterodimers, smaller $\Delta E_{\text {orb }}$ values of -175.3 and $-293.0 \mathrm{kcal} / \mathrm{mol}$ are obtained for the bowl-in-bowl dimers 3 and 4 , respectively, compared to -415.6 and $-460.2 \mathrm{kcal} / \mathrm{mol}$ calculated for the staggered dimers $\mathbf{5}$ and $\mathbf{6}$. These trends show a smaller orbital interaction in the bowl-in-bowl arrangements governed by delocalized $\pi-\pi$ interactions, and indicate a large $\Delta E_{\text {orb }}$ in the staggered dispositions due to the formation of discrete and strong $\mathrm{CH}-\pi$ and S-mediated forces. Note that heterodimers 4 and 6, in which the dithiole rings interact with the hemifullerene, provide larger $\Delta E_{\text {orb }}$ values as expected from the extended electron density of sulfur atoms.

Table 1. Interaction energies $(\mathrm{kcal} / \mathrm{mol}$ ) calculated at the PBEO-D3 with the cc-pVTZ basis set, and with the 6-31G** basis set using several levels of the counterpoise (CP) correction, integrated $\mathrm{NCl}$ volumes $\left(\mathrm{V}_{\mathrm{T}}, \mathrm{Bohr} \mathrm{r}^{3}\right)$, and orbital interaction energies $\left(\Delta E_{\text {orb }}, \mathrm{kcal} / \mathrm{mol}\right)$ as obtained from the NOCV decomposition analysis.

\begin{tabular}{rcrrrrrr}
\hline & $E_{\text {int }}(\mathrm{cc}-\mathrm{pVTZ})$ & \multicolumn{4}{c}{$E_{\text {int }}\left(6-31 \mathrm{G}^{* *}\right)$} & \multirow{2}{*}{$\mathrm{V}_{\mathrm{T}}{ }^{a}$} & $\Delta E_{\text {orb }}$ \\
\cline { 3 - 6 } & & No CP & $\mathrm{CP}$ & $1 / 2 \mathrm{CP}$ & & \\
\hline homodimer 1 & -21.27 & -24.99 & -17.86 & -21.43 & 89.46 & -189.79 \\
homodimer 2 & -22.64 & -26.68 & -21.69 & -24.19 & 77.13 & -462.59 \\
heterodimer 3 & -21.41 & -24.50 & -17.99 & -21.25 & 33.59 & -175.29 \\
heterodimer 4 & -22.00 & -24.98 & -18.57 & -21.78 & 27.36 & -293.03 \\
heterodimer 5 & -24.24 & -28.03 & -22.46 & -25.25 & 31.21 & -415.55 \\
heterodimer 6 & -27.35 & -31.68 & -26.07 & -28.88 & 25.35 & -460.17 \\
\hline
\end{tabular}

${ }^{a}$ A grid step of $0.05 \AA$ was taken along each direction, with cutoffs of 0.03 a.u. and 2.0 a.u. in electron density $\rho$ and reduced density gradient $s$, respectively.

The interaction energy $\left(E_{\text {int }}\right)$ in the hemifullerene dimers was estimated at the PBE0-D3/cc-pVTZ level of theory (Table 1). Both homodimers and heterodimers 1-6 are calculated with an $E_{\text {int }}$ in the range from -21 to $-28 \mathrm{kcal} / \mathrm{mol}$. For homodimers, the staggered arrangement $\mathbf{2}$ is calculated slightly more stable than the bowl-in-bowl structure 1 , with $E_{\text {int }}=-22.64$ and $-21.27 \mathrm{kcal} / \mathrm{mol}$, respectively. This is in agreement with the results previously found for benzene, where $\sigma$-stacking provides a slightly more stable T-type dimer than the $\pi-\pi$ dominated PD dimer. ${ }^{41}$ In the same line, the localized $\mathrm{CH}-\pi$ and S-mediated interactions lead to staggered arrangements 5 and $\mathbf{6}$ being more stable than the $\pi-\pi$ dominated bowl-in-bowl dispositions $\mathbf{3}$ and $\mathbf{4}$ for truxTTF-hemifullerene heterodimers. Heterodimer 6 is calculated to be the most stable assembly with an $E_{\text {int }}=-27.35$ $\mathrm{kcal} / \mathrm{mol}$. These trends are in good accord with the values computed at the revPBE0-D3/cc-pVTZ level, ${ }^{26}$ and with those recently reported using high-level DLPNO-CCSD(T) calculations. ${ }^{27}$ 
In order to have a workable (low computational cost) approach to comprehensively analyze the hemifullerene-based aggregates and to study the effect of the molecular curvature in the assembly (see below), we compared the interaction energies calculated using the $6-31 \mathrm{G}^{* *}$ basis set, both uncorrected and counterpoise (CP)-corrected, with the nearly basis set superposition error (BSSE)-free results obtained with the Dunning's cc-pVTZ basis set (Table 1). Uncorrected interaction energies using the $6-31 G^{* *}$ basis set are significantly overestimated compared to the cc-pVTZ values for the series of hemifullerene-based dimers 1-6. Otherwise, CP-corrected 6-31G** interaction energies tend to underbind the complexes compared to cc-pVTZ. By half-correcting the 6-31G** interaction energies with $\mathrm{CP}$, as done in previous works with much success, ${ }^{42}$ values very close to the cc-pVTZ reference are obtained (Table 1). We therefore consider the PBEO-D3/6$31 \mathrm{G}^{* *}+1 / 2 \mathrm{CP}$ level of theory as a 'workable' approach for the exploratory analysis of the hemifullerene-based dimers with variable curvature detailed in the next sections.

\subsection{Bending the Hemifullerene}

The hemifullerene structure possesses a characteristic bowl shape due to the insertion of fivemember rings in its $\pi$-conjugated structure. Interestingly, after optimizing its geometry by imposing $C_{S}$-symmetry, a completely planar $\pi$-conjugated structure is calculated as a transition state with one imaginary frequency of $125.18 \mathrm{~cm}^{-1}$. The vibrational mode associated to this imaginary frequency connects the two minimum-energy structure wells (mirror images or enantiomers) through the lowest energy path. The intrinsic reaction coordinate (IRC) path from the planar hemifullerene (transition state) to one of the curved structure wells was used to generate hemifullerene structures of increasing curvature (see Computational Details). The IRC calculation generated 30 structures, from which 15 geometries equally distributed along the path were extracted. For simplicity, two indexes $(X, Y)$ are introduced to refer to the degree of curvature of the top $(X)$ and bottom $(Y)$ moieties in the hemifullerene homodimer ranging from 0 (planar) to 14 (highly curved) as defined in Figure 4 . Note that the structure with index 14 corresponds to the minimum-energy geometry of hemifullerene. 


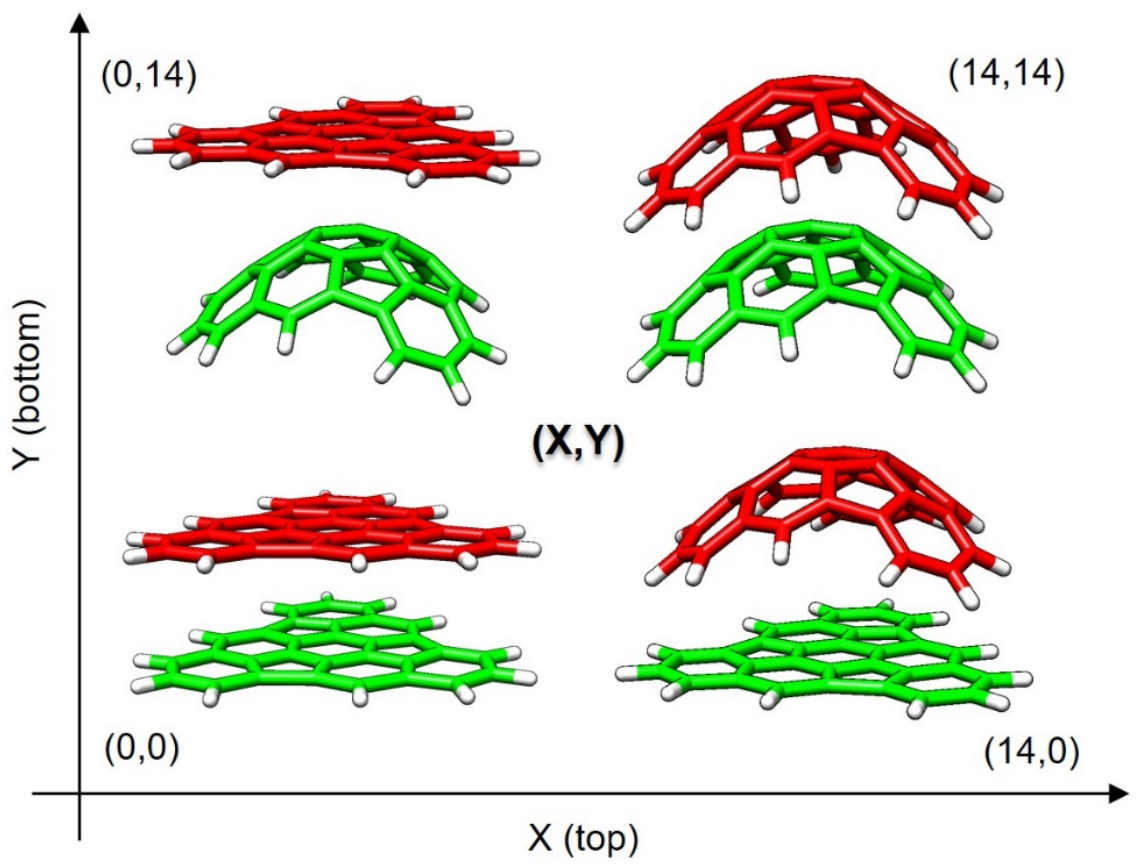

Figure 4. Schematic representation of the extreme dispositions for the bowl-in-bowl hemifullerene homodimer with variable curvature. $X(Y)=0$ indicates a completely planar hemifullerene structure, whereas $X(Y)=14$ refers to the most curved arrangement.
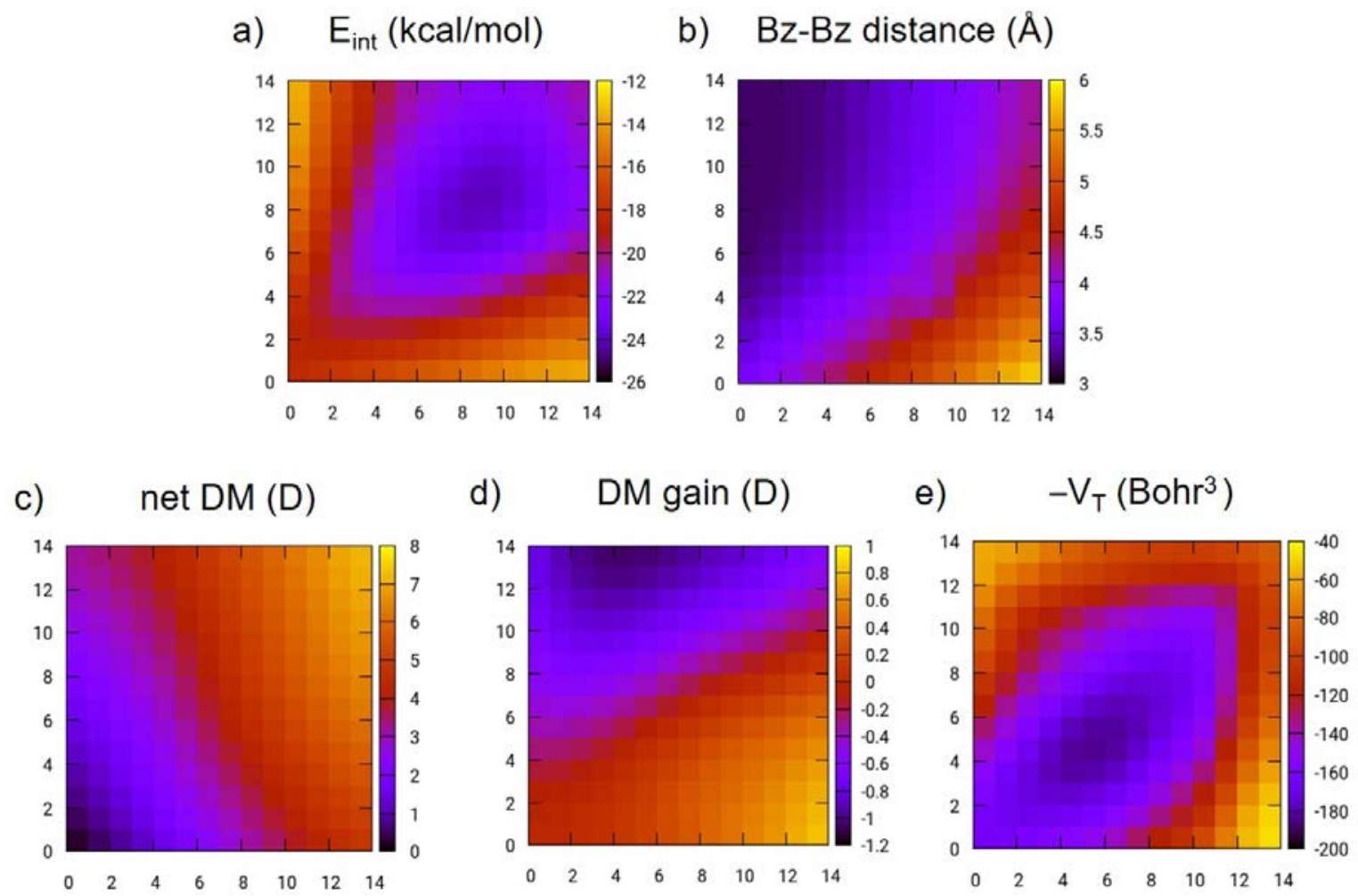

Figure 5. Energy, geometry, electronic and integrated $\mathrm{NCl}$ properties calculated along the 2D curvature scan for the bowl-in-bowl homodimer 1. $x$ and left- $y$ axes represent the $X$ and $Y$ indexes, 
respectively, defining the curvature of the hemifullerene moieties forming the dimer. The values of the magnitude specified on the top are represented on the graphics following the color scale given on right- $y$ axis. a) Interaction energy $\left(E_{\text {int }}\right)$ calculated at the PBE0-D3/6-31G**+1/2CP level. b) Intermolecular distance between the centroids of the central benzene (Bz) rings. c) Net dipole moment (DM). d) Dipole moment gain in the dimer with respect to the sum of the dipole moments calculated for the constituting monomers at the geometry of the dimer. e) Integrated volumes $\left(\mathrm{V}_{T}\right)$ along the $\mathrm{NCl}$ surface (with negative sign).

Figure 5 shows the evolution of several electronic properties and geometry parameters calculated for the supramolecular hemifullerene bowl-in-bowl homodimer 1 upon increasing the curvature of both top and bottom monomers as shown in Figure 4. The scan along the curvature was performed with rigid intramolecular geometrical parameters and only optimizing the intermolecular distance between fragments (the relative intermolecular orientation of the two monomers was kept fixed).

The interaction energy calculated at the PBE0-D3/6-31G**+1/2CP level starting from a completely planar-planar dimer $(0,0)$ is shown to increase upon increasing simultaneously the curvature of both fragments (diagonal line, Figure 5a). The maximum value of $E_{\text {int }}$ is calculated at -24.28 $\mathrm{kcal} / \mathrm{mol}$ for the bowl-in-bowl homodimer with curvature $(9,9)$. Otherwise, if only one fragment is curved ( $x$ or $y$-axis), the interaction energy decreases since the arrangement becomes less efficient. The evolution of $E_{\text {int }}$ is directly correlated with the dispersion D3 correction indicating that the assembly is mainly driven by weak noncovalent interactions. The second version of the Grimme's atom-pairwise dispersion correction (D2) was also assessed along the scan (Figure S4a). The D2 dispersion coefficients do not take into account the environment (coordination) of a particular atom, and therefore the intermolecular D2 stabilization is an indirect measure of the overall proximity of one hemifullerene with respect to the other. In this sense, the point with maximum interaction energy $(9,9)$ corresponds with the one with a larger intermolecular D2 term, and thus with a dimer in which the two hemifullerene monomers are closest between each other (maximum concave-convex complementarity). The same trend is observed for the intermolecular nuclear repulsion term (Figure S4b).

The distance between the centroids of the central benzene rings ( $\mathrm{Bz}-\mathrm{Bz}$ distance) was also monitored along the scan (Figure 5b). Overall, the central benzenes are closest in the $(0,14)$ geometry, where the top fragment is planar and interacts with the convex region of the highly curved bottom moiety (see Figure 4). Along the diagonal line, the Bz-Bz distance does not vary much, with slightly larger values computed upon increasing the curvature of both fragments.

Interestingly, the net dipole moment (DM) increases upon increasing the curvature of the fragments, either independently or simultaneously (Figure 5c). The most curved $(14,14)$ dimer provides the largest dipole moment (7.24 D) among the 2D scan and suggests that dipole-dipole interactions may have a stabilizing role, as demonstrated in recent works on coronene and corannulene. ${ }^{25} \mathrm{By}$ analyzing the DM gain in the dimer with respect to the sum of the dipole moments calculated for the constituting monomers at the geometry of the dimer (Figure $5 \mathrm{~d}$ ), interesting trends are found. The major gain in DM is obtained for dimer $(14,0)$ where the concave part of the top hemifullerene interacts with the completely planar bottom hemifullerene (Figure 4). On the contrary, the DM gain in the opposite site region $(0,14)$ turns out to be negative, which 
means that the DM in the dimer is lower than in the constituting monomers. These two scenarios stem from the different behavior of the concave and convex regions in the curved fragment with respect to the planar fragment. Whereas the concave region is able to pull electron density from the planar fragment allowing an increase in the total DM, the planar fragment on top of the convex surface of the other moiety acts as a shield, attenuating the DM generated by the curved fragment. The DM gain along the diagonal line is practically zero indicating that the DM of the resulting dimer is the sum of the DMs of the constituting hemifullerene structures (Figure $5 \mathrm{~d}$ ).

Finally, the integrated $\mathrm{NCl}$ volumes were calculated along the $2 \mathrm{D}$ scan (Figure $5 \mathrm{e}$ ) with a grid step of $0.10 \AA$ along each direction, and cutoffs of 0.02 a.u. and 2.0 a.u. in electron density $\rho$ and reduced density gradient $s$, respectively. As a whole, the maximum value calculated for the integrated total volume $\left(\mathrm{V}_{\mathrm{T}}=185.61\right.$ a.u. $)$ is found close to the diagonal line $(5,4)$, providing a similar image to $E_{\text {int }}$. However, the volume is maximized at shorter curvatures than the point with maximum interaction energy $(9,9)$. This recalls that, like in Atoms in Molecules theory, the total interaction energy is not only governed by the amount of $\mathrm{NCl}$ interactions along the intermolecular region but also by the nature and strength of these forces $(\mathrm{CH}-\pi, \pi-\pi$, dipolar, etc.). The $\mathrm{NCl}$ volume can provide an indicative of the geometric arrangement (and thus, an indirect proof of dispersion), but only when the main nature of the interactions does not change. Dipole-dipole forces, not taken into account by the integrated $\mathrm{NCl}$ volume, start to become relevant upon increasing the curvature and explain why the point of maximum interaction energy shifts to larger-curved dimers.
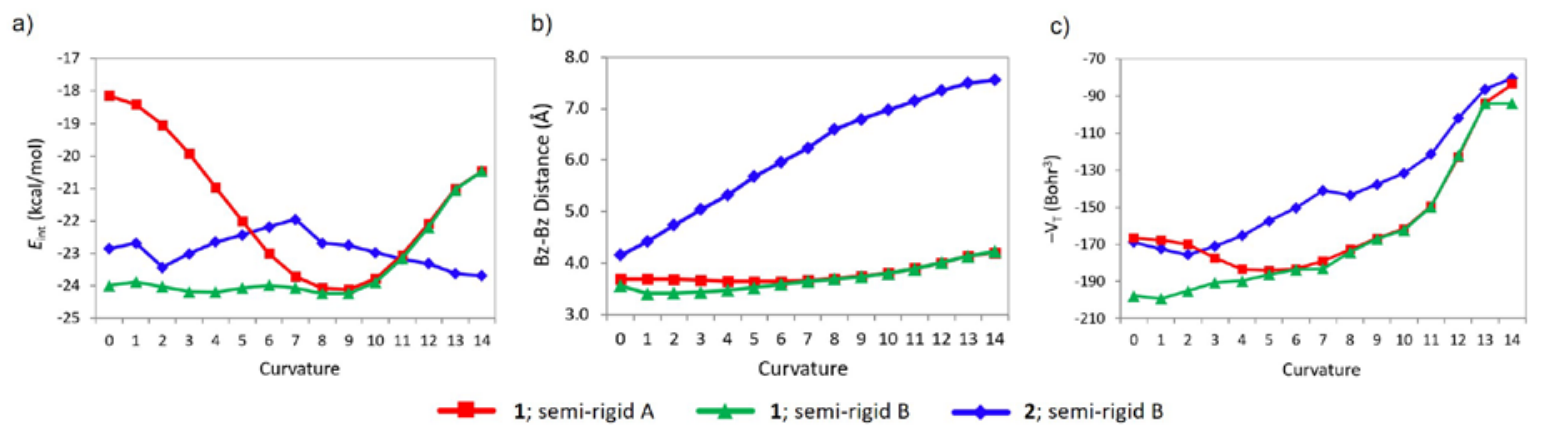

Figure 6. Evolution of the interaction energy (a), the intermolecular Bz-Bz distance (b), and the integrated volume $\left(\mathrm{V}_{\mathrm{T}}\right)$ in the $\mathrm{NCl}$ region (c) for two levels of semi-rigid optimizations of homodimers 1 and $\mathbf{2}$ by varying the curvature of both hemifullerene moieties at the same time ( $X$ $=Y$ ). In the semi-rigid optimization $A$, only the intermolecular distance is allowed to change, whereas, in the semi-rigid procedure $\mathrm{B}$, all the intermolecular geometry parameters are optimized.

A deeper insight into the assembly of both bowl-in-bowl and staggered homodimers of hemifullerene was undertook by varying the curvature of both $\mathrm{C}_{30} \mathrm{H}_{12}$ moieties at the same time $(X$ $=Y$, Figure 6). Two levels of geometry optimization were performed: a semi-rigid procedure $A$, in which only the intermolecular distance between the two moieties is optimized, and a semi-rigid protocol B, where all the intermolecular coordinates were relaxed. Note that protocol A is not applied to homodimer $\mathbf{2}$ since it would not allow displacement of one moiety over the other to generate staggered dispositions. As shown in Figure 6a, the maximum interaction energy 
computed for the bowl-in-bowl homodimer 1 using protocol A is obtained for an intermediate situation $(X=9)$ closer to the more curved dimers, as already inferred from Figure 5a. However, by allowing the dimers to optimize the relative orientation of one hemifullerene with respect to the other (protocol B), low-curved and planar dimers are computed to be more stable compared to level $A$, obtaining a nearly flat interaction energy profile from $X=0$ to $X=9$ (Figure 6a). Moving to the staggered dimer $\mathbf{2}$, a completely different profile is computed upon increasing the curvature of the system, obtaining maximum interaction energies at the edges of the scan for planar-planar and largely curved-curved dimers, whereas in the intermediate region $(X=7)$ a maximum with the lowest interaction energy $\left(E_{\text {int }}=-22.0 \mathrm{kcal} / \mathrm{mol}\right)$ is calculated. Note that $E_{\text {int }}$ does not coincide in semi-rigid $B$ optimizations of $\mathbf{1}$ and $\mathbf{2}$ for planar homodimers $(X=0)$ due to the presence of several local minima. Interestingly, different trends are predicted in $E_{\text {int }}$ for $\mathbf{1}$ and $\mathbf{2}$ when using the semirigid optimization protocol B (Figure 6a): bowl-in-bowl dimer 1 provides larger interaction energies for low-curved buckybowls (from $X=2$ to 9), whereas the staggered dimer $\mathbf{2}$ is more stabilized for highly-curved fullerene fragments (from $X=9$ to 14). This result is particularly interesting since different types of supramolecular assemblies (bowl-in-bowl or staggered) may be accomplished experimentally by tuning the curvature of the carbon-based nanoform.

The $\mathrm{Bz}-\mathrm{Bz}$ distance between the central benzene rings was tracked along the curvature $(X=Y)$ scans for the bowl-in-bowl and staggered dimers (Figure 6b). Whereas in 1, the Bz-Bz distance does not vary much upon increasing the curvature of the hemifullerene, this parameter enlarges from ca. 4.0 to $7.5 \AA$ in the staggered dimer 2. For the latter, the curvature of the hemifullerene fragment forces the dimer to acquire an arrangement in which, although the central benzenes move away from each other, the two monomers are efficiently interlocked favoring the total stabilization of the complex.

Figure $6 \mathrm{c}$ shows the evolution of the integrated $\mathrm{NCl}$ volumes calculated for the two hemifullerene dimers in going from planar-planar to curved-curved dimers. The semi-rigid profile A calculated for the bowl-in-bowl homodimer $\mathbf{1}$ is extracted from the diagonal line of the plots shown in Figure 5 , and exhibits $\mathrm{V}_{T}$ maximum $\left(-\mathrm{V}_{\mathrm{T}}\right.$ minimum) at $X=5$. Moving to the more flexible scan $\mathrm{B}$, the integrated volumes deviate from the profiles obtained in protocol A for flat-flat dimers $(X<7)$. The integrated $\mathrm{NCl}$ volume tends to decrease upon increasing the curvature of the hemifullerene in scan B. The larger values of the integrated $\mathrm{NCl}$ properties calculated for scan B are indicative of the more efficiently interacting assemblies formed. For the staggered homodimer $\mathbf{2}$, the integrated $\mathrm{NCl}$ properties also decrease going from flat to curved fragments, showing a low peak computed at $X=7$ that corresponds to the local maximum found in the $E_{\text {int }}$ profile (Figure 6a). Although larger $\mathrm{NCl}$ volumes are generally calculated for flatter hemifullerene dimers, the total interaction energy is governed by both the amount and intensity of the noncovalent interactions driving the assembly. Therefore, as we have seen with the $\mathrm{NCl}$ analysis, the intensity rather than the amount of $\mathrm{NCl}$ interactions prevails in curved dimers, leading to more stable assemblies compared to the bowl-in-bowl analogues. 


\subsection{LOLIPOP Index}

The number of supramolecular complexes involving buckyballs and buckybowls through $\pi-\pi$ interactions reported in the literature is scarce. The inefficiency of these curved carbon nanoforms to interact through $\pi-\pi$ forces is however ascribed to a geometric effect rather than to an electronic reason. ${ }^{25}$ In fact, the strength of the binding in the prominent buckycatcher $\cdot \mathrm{C}_{60}$ complex is believed to come from "pure" $\pi-\pi$ interactions through an adequate concave-convex complementarity. ${ }^{13}$

$\pi$-depletion has been recently proposed as a design principle to elucidate the strength of conjugated molecules to $\pi$-stack: the bigger the $\pi$-electron depletion, the stronger the $\pi$-stacking ability. ${ }^{35}$ In this regard, Corminboeuf et al. have introduced a quantitative criterion, so-called LOLIPOP, based on the Localized Orbital Locator (LOL), which offers straightforward insight into the $\pi$-electron depletion in aromatic organic molecules. ${ }^{35}$

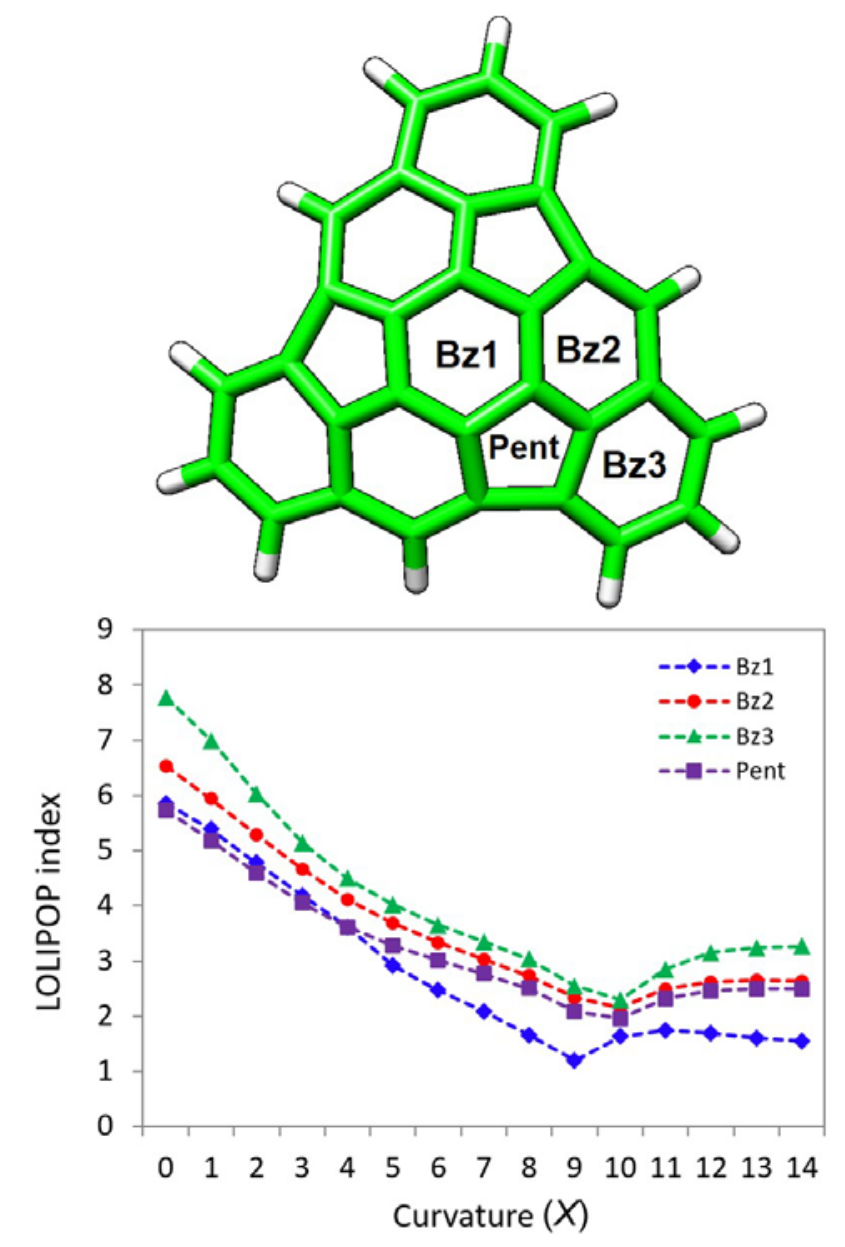

Figure 7. LOLIPOP index for selected 6- and 5-member rings of hemifullerene as a function of curvature $(X)$.

The LOLIPOP index was estimated for the hemifullerene fragment as a function of the molecular curvature. The LOL function was calculated for the $\pi$-density $\left(\mathrm{LOL}_{\pi}\right)$ and integrated for LOL values larger than 0.55 over a cylinder of radius $1.94 \AA$ centered in specific rings of the fragment, and 
extended up to $0.5 \AA$ from the ring plane. Figure 7 shows the evolution of LOLIPOP values calculated for 6 - and 5-member rings of the hemifullerene monomer upon increasing its curvature. The LOLIPOP index decreases going from the planar structure $(X=0)$ to more curved geometries for all the rings analyzed, and reaches minimum values at curvatures corresponding to $X=9$ and 10. The lowest LOLIPOP value for the central benzene ring (Bz1 in Figure 7) is calculated to be 1.20 at $X=9$, whereas it is predicted slightly higher in the range of 1.95-2.30 for the rest of 6- and 5member rings at a curvature of $X=10$. Interestingly, these curvatures of maximum $\pi$-depletion correspond with the maximum interaction energy calculated for the bowl-in-bowl homodimer 1 (see Figure 5 and 6). A further increase of the hemifullerene curvature from $X=9 / 10$ to $X=14$ is not translated into larger $\pi$-depletion, and LOLIPOP index slightly increases (Figure 7). The trends in the LOLIPOP index were preserved after small variations of the cylinder radius probe in $\pm 0.20 \AA$ (Figure S5 and S6).

Figure 8 displays the $\mathrm{LO}_{\pi}$ isosurfaces for three representative hemifullerene structures $(X=0,9$, and 14). Whereas large $L^{2} L_{\pi}$ surfaces are computed for planar hemifullerene $(X=0)$, a decrease in the $\mathrm{LOL}_{\pi}$ surface is obtained upon bending. The smallest $\mathrm{LOL}_{\pi}$ surface is shown by structure $X=10$. For more curved structures $(X>10)$, the $\mathrm{LOL}_{\pi}$ slightly increases and stabilizes up to the most curved dimer $X=14$ (Figure 8 ).
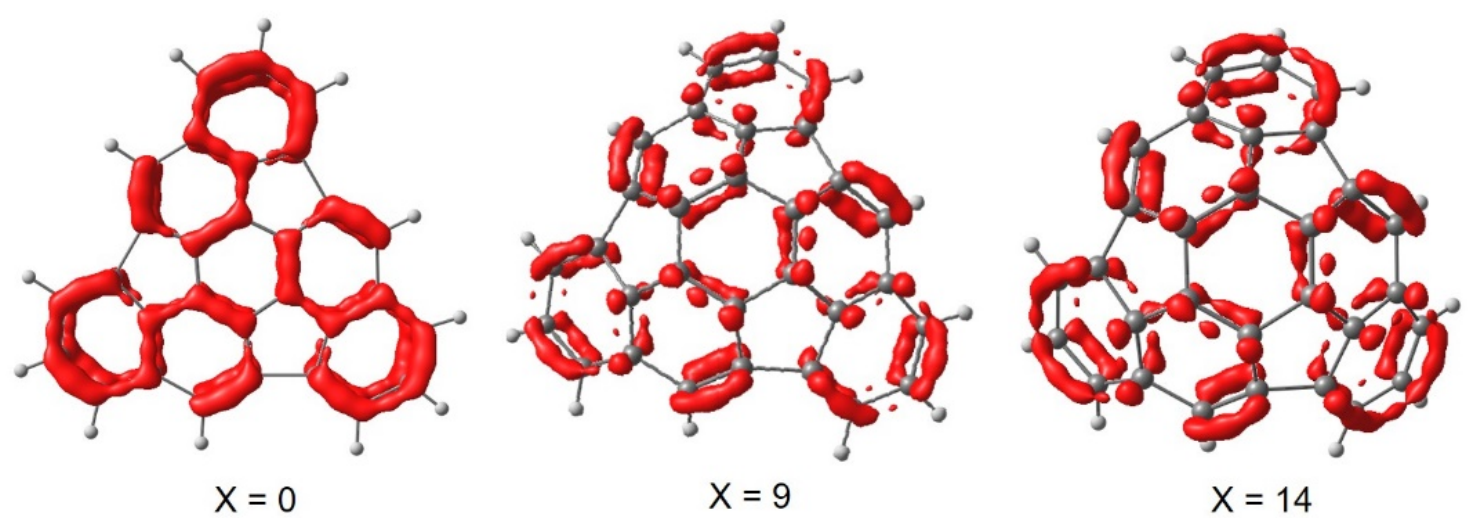

Figure 8. $L O L_{\pi}$ isosurfaces (isovalue $=0.55$ ) for three representative hemifullerene structures going from flat $(X=0)$ to curved $(X=14)$ geometries.

The LOLIPOP index therefore provides valuable information on the $\pi$-stacking ability of hemifullerene upon changing its curvature, showing that for relatively largely curved geometries, the $\pi$-depletion is large (low values of LOLIPOP) and the $\pi$ stacking is enhanced. This feature is particularly important in bowl-in-bowl assemblies governed by $\pi-\pi$ stacking, as is the case of homodimer $\mathbf{1}$ and heterodimers $\mathbf{3}$ and $\mathbf{4}$. However, we predict a curvature for which the $\pi$-stack efficiency is maximum (minimum LOLIPOP values at $X=9 / 10$ ), indicating that an increase of the curvature from then on would no longer enhance the $\pi$-stacking ability to reach more stable supramolecular bowl-in-bowl associates. This idea is in good accord with the evolution predicted for the interaction energy of the bowl-in-bowl homodimer $\mathbf{1}$ (red profile in Figure 6a). 


\subsection{Curvature Effect on the TruxTTF-Hemifullerene Recognition}

The list of hemifullerene structures with variable bending ( $X=0$ to 14 ) was finally used to unravel the effect of the buckybowl curvature in the association with the electron-donor truxTTF molecule. Minimum-energy geometries were calculated for the different heterodimers keeping frozen the relative intramolecular atomic positions and only varying the characteristic intermolecular parameters of the assembly. Figures 9a and 10a display the interaction energy calculated at the PBE0-D3/6-31G**+1/2CP level of theory, whereas Figure 11 summarizes the intermolecular distance between the central benzene rings in each heterodimer.

In bowl-in-bowl heterodimers $\mathbf{3}$ and $\mathbf{4}$, an increase in the hemifullerene curvature correlates with a decrease in the intermolecular distance between the central rings of each moiety (Figure 11). However, this is not directly translated into a larger interaction energy. For heterodimer $\mathbf{3}$, the maximum $E_{\text {int }}$ is computed for the hemifullerene with a relatively small curvature $(X=4$; Figure 9 a left). The convex region of this structure fits into the carbon-based concave skeleton of truxTTF for a maximum interaction $(-23.80 \mathrm{kcal} / \mathrm{mol})$ through $\pi-\pi$ contacts calculated in the range of 3.3-3.5 $\AA$ (Figure 9b left). On the contrary, the dithiol concave region of truxTTF has a considerably larger curvature and hemifullerene structures with large bendings provide the highest $E_{\text {int }}$ values (Figure 9a-right and 9b-right). The maximum interaction energy for bowl-in-bowl heterodimer 4 is calculated at $X=10$ with an $E_{\text {int }}=-18.23 \mathrm{kcal} / \mathrm{mol}$, originated from close $\pi-\pi(3.3-4.1 \AA)$ and S $-\pi$ (3.55 $\AA$ ) contacts (Figure 9b right). The most efficient curvature for hemifullerene in bowl-in-bowl arrangements therefore depends on the curvature of the host concave region of the truxTTF to which it interacts.
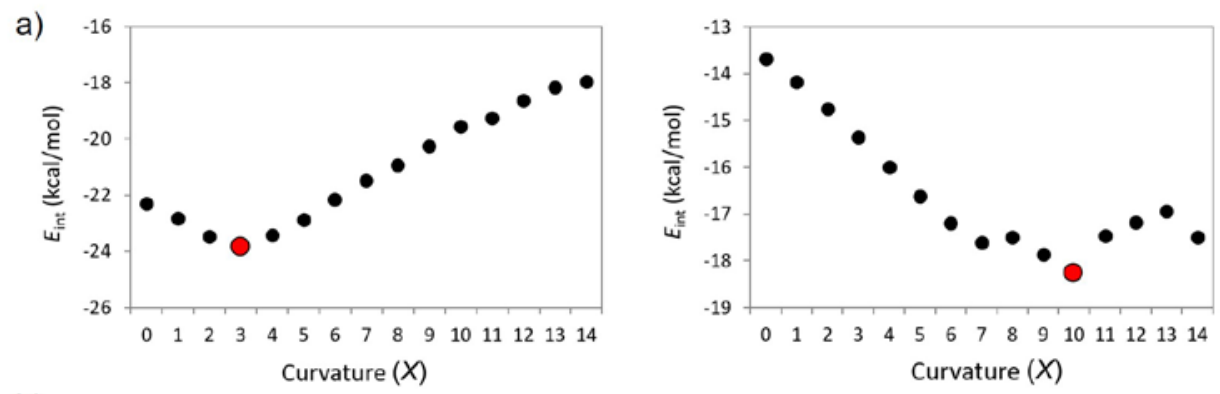

b)
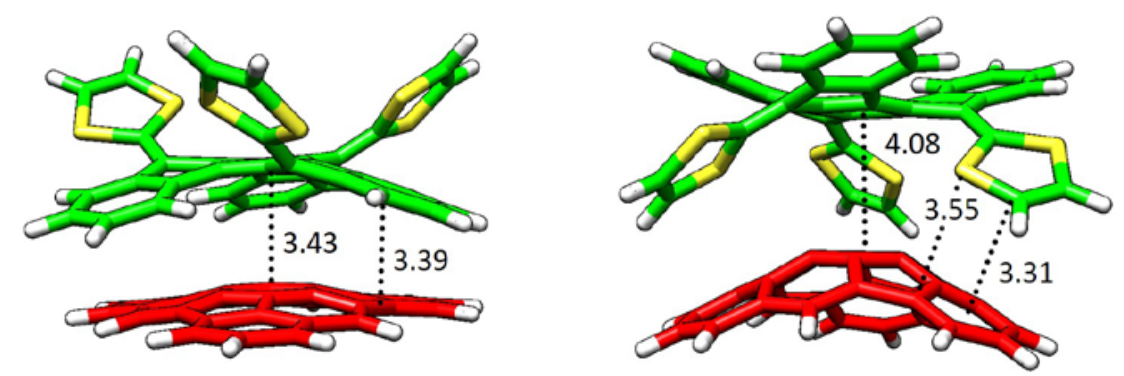

Figure 9. a) Interaction energy for heterodimers 3 (left) and 4 (right) as a function of the hemifullerene curvature $(X)$. Points corresponding to the minimum-energy structures calculated with the largest interaction energy for each heterodimer are highlighted in red. b) Minimumenergy structure calculated with the largest interaction energy for heterodimer $\mathbf{3}$ (left) and $\mathbf{4}$ (right). 
Moving to the staggered heterodimers, the interaction energy profile by varying the hemifullerene curvature is flatter compared to the bowl-in-bowl arrangements, especially for $\mathbf{5}$. In this dimer, a large $E_{\text {int }}$ of $-23 \mathrm{kcal} / \mathrm{mol}$ is predicted starting from the planar hemifullerene $(X=0)$, due to efficient $\pi-\pi$ interactions calculated in the range of 3.2-3.8 $\AA$. By increasing the buckybowl curvature, hemifullerene moves away from the aromatic truxene core to accommodate one of the peripheral benzene rings of truxTTF in its concave region (Figure 11). Calculations predict the largest interaction energy of $-24.33 \mathrm{kcal} / \mathrm{mol}$ for heterodimer 5 with a hemifullerene curvature of $X=13$, with efficient $\pi-\pi(3.6-3.8 \AA)$ and $\mathrm{CH}-\pi(2.5-2.7 \AA)$ interactions (Figure 10b left).

The dithiol face of truxTTF does not benefit from interacting with a planar surface as shown in the $E_{\text {int }}$ profile calculated for the staggered heterodimer 6 (Figure 10a right). Due to the highly curved concave dithiole region of truxTTF, flat hemifullerenes are not adequate, and interaction energies smaller than $-23 \mathrm{kcal} / \mathrm{mol}$ are predicted in the association. However, bending the hemifullerene buckybowl results in a displacement of the carbon nanoform towards one peripheral dithiol ring (see Figure 11 for the $\mathrm{Bz}-\mathrm{Bz}$ distance), for which larger interaction energies are calculated. The largest $E_{\text {int }}$ value $(-27.85 \mathrm{kcal} / \mathrm{mol})$ is predicted for a hemifullerene curvature of $X=10$, for which a combination of efficient $\pi-\pi(3.5-3.7 \AA), \mathrm{CH}-\pi(2.5-2.7 \AA)$, and S-mediated (3.5 $)$ interactions are present (Figure 10b right).
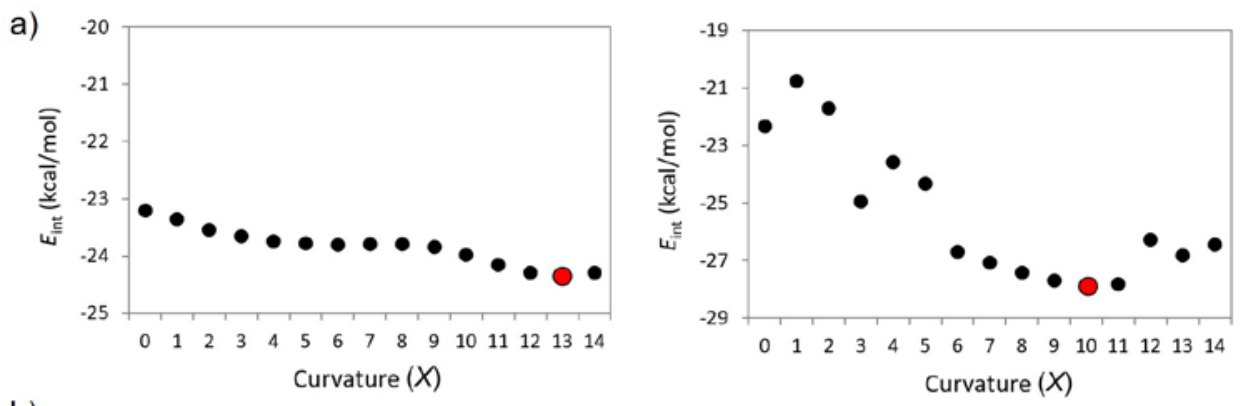

b)
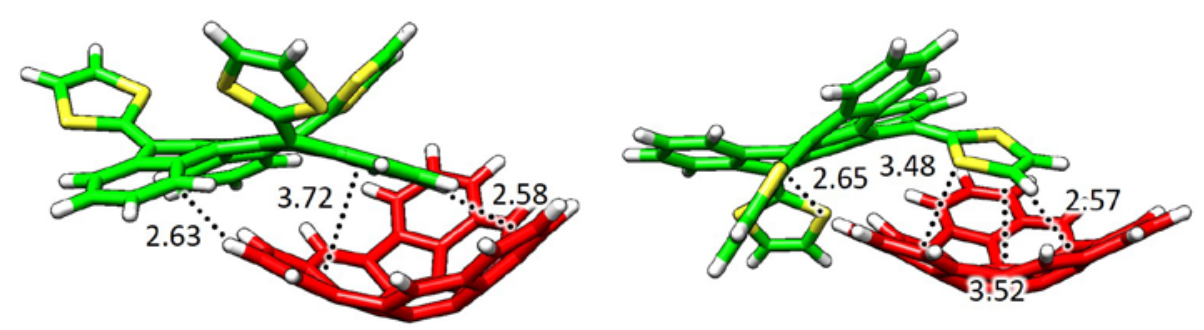

Figure 10. a) Interaction energy for heterodimers 5 (left) and 6 (right) as a function of the hemifullerene curvature $(X)$. Points corresponding to the minimum-energy structures calculated with the largest interaction energy for each heterodimer are highlighted in red. b) Minimumenergy structure calculated with the largest binding energy for heterodimer $\mathbf{5}$ (left) and $\mathbf{6}$ (right). 


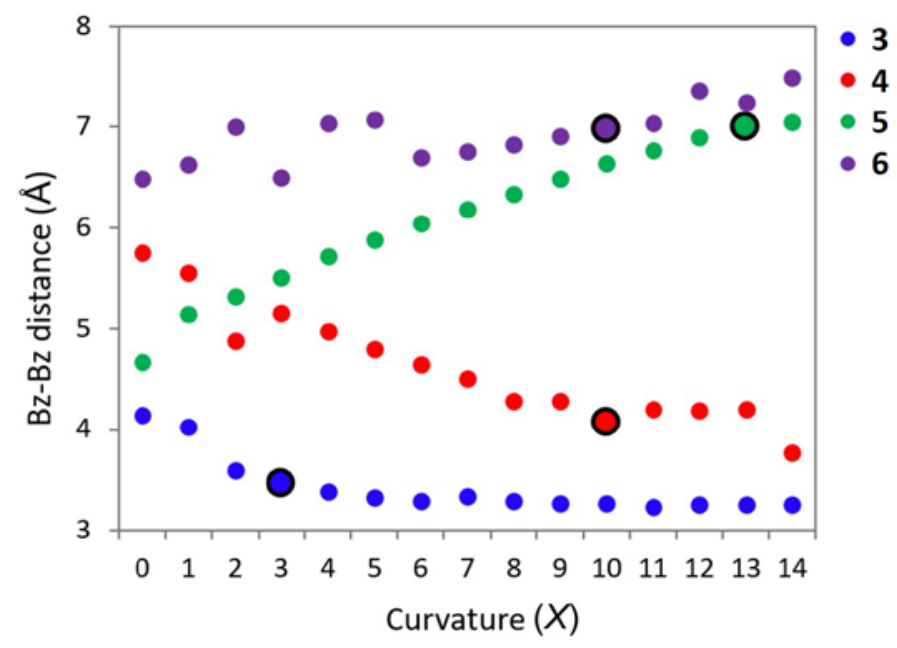

Figure 11. Evolution of the distance between the centroids of the central benzene rings of truxTTF and $\mathrm{C}_{30} \mathrm{H}_{12}$ as a function of the curvature $(X)$ of the fullerene fragment in the optimized assembly (intramolecular geometry parameters are frozen during the optimization). Points corresponding to the minimum-energy structures calculated with the largest interaction energy for each heterodimer are highlighted with black stroke.

Finally, the net dipole moment (DM) for the different heterodimers upon increasing the hemifullerene curvature was analyzed (Figure 12). The minimum-energy structure of hemifullerene presents a dipole moment calculated to be $3.93 \mathrm{D}$ pointing from the concave to the convex region, whereas the donor truxTTF molecule is predicted with a DM of 4.28 D pointing from the dithiol region to the carbon-based skeleton (Figure 12a). 
a)

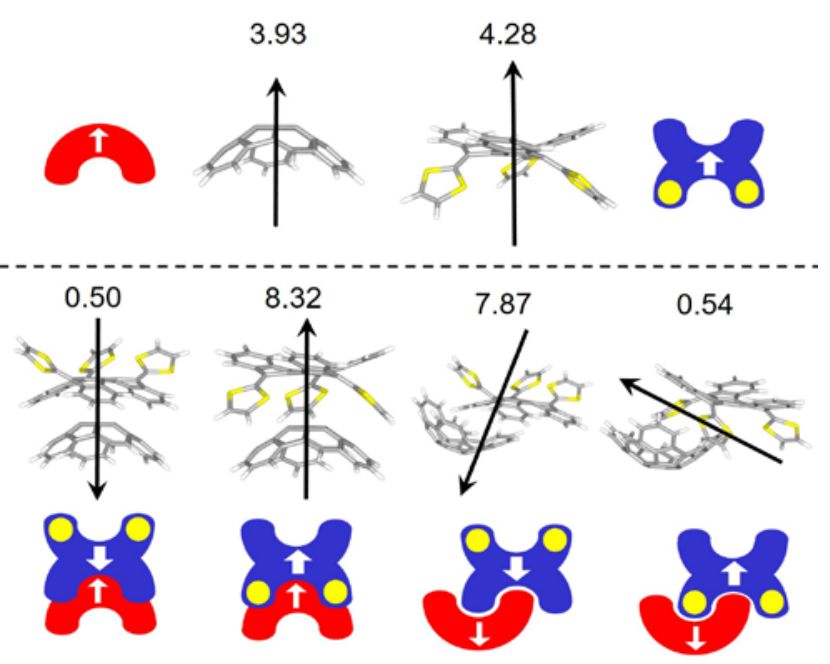

b)

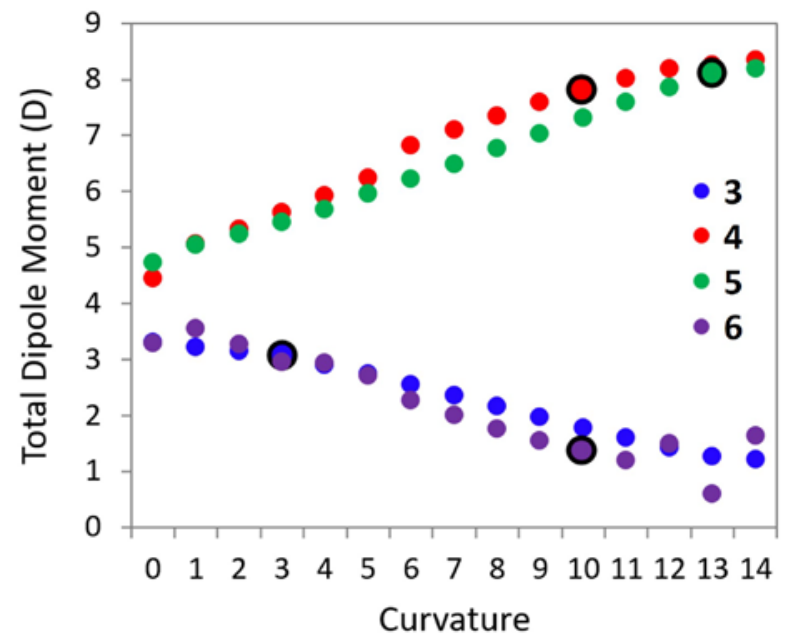

Figure 12. a) Representation of the net dipole moment calculated for the four possible arrangements 3-6 of the truxTTF. $\mathrm{C}_{30} \mathrm{H}_{12}$ dimer (bottom) and the constituting monomers (top). The value of the dipole moment (in D) is quoted. b) Evolution of the dipole moment for 3-6 as a function of the hemifullerene curvature $(X)$. Points corresponding to the minimum-energy structures calculated with the largest interaction energy for each heterodimer are highlighted with black stroke.

The evolution of the total DM in the different heterodimers (Figure 12b) can be easily understood by taking into account the DM of the constituting monomers individually (Figure 12a). In heterodimers 3 and $\mathbf{6}$, the DM of truxTTF and $\mathrm{C}_{30} \mathrm{H}_{12}$ are oriented oppositely; thus, upon increasing the hemifullerene curvature, the total DM of the heterodimer diminishes. In contrast, the DM of the constituting monomers in heterodimers $\mathbf{4}$ and $\mathbf{5}$ are parallel oriented, and an increase in the buckybowl curvature is translated into an increase of the total DM (Figure 12b). According to the results reported by Sherrill et al., ${ }^{25}$ an increase in the buckybowl curvature leads to a larger dipole moment and to an enhanced intermolecular electrostatic interaction in concave-convex arrangements. In this regard, heterodimer 4, with aligned DMs for its constituting moieties, is calculated with a larger interaction energy compared with the bowl-in-bowl analogue 3 (Table 1). 
However, this reasoning no longer holds when comparing 5 and $\mathbf{6}$. Staggered arrangements are far more complex than bowl-in-bowl dispositions, showing a combination of different types of noncovalent interactions and local electrostatic forces originating from the intercalation of one moiety into the other.

\section{CONCLUSIONS}

A thorough theoretical characterization of bowl-in-bowl (concave-convex) and staggered (concave-concave) supramolecular homodimers of hemifullerene $\mathrm{C}_{30} \mathrm{H}_{12}$ and heterodimers of $\mathrm{C}_{30} \mathrm{H}_{12}$ with the electron-donor truxTTF system is presented. Special attention was paid to unravel the origin and nature of the forces governing the concave-concave arrangement present in the staggered dimers, for which detailed information is missing so far. Our results through chemical bonding techniques confirm a complex $\mathrm{NCl}$ signature with the presence of discrete and strong forces stabilizing the formation of the staggered dimers, accompanied by an enhanced orbital interaction, compared with the $\pi-\pi$ dominated bowl-in-bowl arrangements. Complementing the $\mathrm{NCl}$ analysis with the electron density values at the intermolecular bond critical points allows to better understand the local interactions in the most stable staggered heterodimers, which come from discrete $\mathrm{CH}-\pi$ and sulfur-mediated ( $\mathrm{S}-\pi$ and $\mathrm{S}-\mathrm{H}$ ) forces.

The analysis of the buckybowl curvature showed that bending the fullerene fragment favors the stabilization of both homo- and heterodimers, in good accord with the depletion in the r-electron density calculated upon increasing curvature. Interestingly, two regimes are predicted for the hemifullerene homodimer: the bowl-in-bowl dimer shows larger interaction energies for lowcurved buckybowls, whereas the staggered arrangement is more stabilized for highly-curved fullerene fragments. This result is particularly interesting since different types of supramolecular assemblies (bowl-in-bowl or staggered) may be accomplished experimentally by tuning the curvature of the carbon-based nanoform.

For the heterodimers, the optimal buckybowl curvature is demonstrated to depend on the type of arrangement (bowl-in-bowl, from medium to small curvatures, vs. staggered, from medium to large curvatures), and on the concave face of the truxTTF donor to which hemifullerene interacts. The dipole moment of the resulting assembly has demonstrated to stabilize the bowl-in-bowl arrangement in which the buckybowl approaches the dithiol concave region of truxTTF. In contrast, dipole-dipole interactions are shown not to correlate with the interaction energy calculated for staggered dispositions. These arrangements maximize the concave-concave coupling by a complex scenario of $\pi-\pi, \mathrm{CH}-\pi$ and S-mediated noncovalent interactions.

The results presented here shed light into the origin of the forces stabilizing unconventional concave-concave staggered supramolecular arrangements involving carbon-based buckybowls, and will help in addressing the development of a new host-guest chemistry dominated by other than the prototypical $\pi-\pi$ concave-convex complementarity. 


\section{ASSOCIATED CONTENT}

Supporting Information. The Supporting Information is available free of charge on the ACS Publications website at DOI: XXX/XXX.XXX.XXX

Figures S1-S4, Tables S1-S6 and XYZ coordinates

\section{AUTHOR INFORMATION}

\section{Corresponding Authors}

* (J.C.) E-mail: joaquin.calbo@uv.es

*(E.O.) E-mail: enrique.orti@uv.es

*(J.C.-G.) E-mail: contrera@lct.jussieu.fr

\section{Notes}

The authors declare no competing financial interest.

\section{ACKNOWLEDGEMENTS}

Financial support from MINECO of Spain (CTQ2015-71154-P, CTQ2015-71936-REDT, and Unidad de Excelencia María de Maeztu MDM-2015-0538), Generalitat Valenciana (PROMETEO/2016/135), and European FEDER funds (CTQ2015-71154-P) is acknowledged. J.C. is grateful to the Generalitat Valenciana for a post-doctoral fellowship (APOSTD/2017/081). J.A. is acknowledged to the MINECO for a "JdC-Incorporación" Fellowship (IJCI-2015-26154).

\section{REFERENCES}

(1) Petrukhina, M. A.; Scott, L. T.; Kroto, H. W., Fragments of Fullerenes and Carbon Nanotubes: Designed Synthesis, Unusual Reactions, and Coordination Chemistry. Wiley: 2011; p 440.

(2) Delgado, J. L.; Bouit, P.-A.; Filippone, S.; Herranz, M. Á.; Martín, N., Organic photovoltaics: a chemical approach. Chem. Commun. 2010, 46, 4853-4865.

(3) Dennler, G.; Scharber, M. C.; Brabec, C. J., Polymer-Fullerene Bulk-Heterojunction Solar Cells. Adv. Mater. 2009, 21, 1323-1338.

(4) Brabec, C. J.; Gowrisanker, S.; Halls, J. J. M.; Laird, D.; Jia, S.; Williams, S. P., Polymer-Fullerene Bulk-Heterojunction Solar Cells. Adv. Mater. 2010, 22, 3839-3856.

(5) Martín, N.; Sánchez, L.; Herranz, M. Á.; Illescas, B.; Guldi, D. M., Electronic Communication in Tetrathiafulvalene (TTF)/C60 Systems: Toward Molecular Solar Energy Conversion Materials? Acc. Chem. Res. 2007, 40, 1015-1024.

(6) Wróbel, D.; Graja, A., Photoinduced electron transfer processes in fullerene-organic chromophore systems. Coord. Chem. Rev. 2011, 255, 2555-2577. 
(7) Fukuzumi, S.; Kojima, T., Photofunctional nanomaterials composed of multiporphyrins and carbon-based pi-electron acceptors. J. Mater. Chem. 2008, 18, 1427-1439.

(8) Imahori, H., Porphyrin-fullerene linked systems as artificial photosynthetic mimics. Org. Biomol. Chem. 2004, 2, 1425-1433.

(9) Petrukhina, M. A.; Scott, L. T., Coordination chemistry of buckybowls: from corannulene to a hemifullerene. Dalton Trans. 2005, 2969-2975.

(10) Mizyed, S.; Georghiou, P. E.; Bancu, M.; Cuadra, B.; Rai, A. K.; Cheng, P.; Scott, L. T., Embracing C60 with Multiarmed Geodesic Partners. J. Am. Chem. Soc. 2001, 123, 12770-12774.

(11) Georghiou, P. E.; Tran, A. H.; Mizyed, S.; Bancu, M.; Scott, L. T., Concave Polyarenes with Sulfide-Linked Flaps and Tentacles: New Electron-Rich Hosts for Fullerenes. J. Org. Chem. 2005, 70, 6158-6163.

(12) Dawe, L. N.; AlHujran, T. A.; Tran, H.-A.; Mercer, J. I.; Jackson, E. A.; Scott, L. T.; Georghiou, P. E., Corannulene and its penta-tert-butyl derivative co-crystallize $1: 1$ with pristine C60-fullerene. Chem. Commun. 2012, 48, 5563-5565.

(13) Sygula, A.; Fronczek, F. R.; Sygula, R.; Rabideau, P. W.; Olmstead, M. M., A Double Concave Hydrocarbon Buckycatcher. J. Am. Chem. Soc. 2007, 129, 3842-3843.

(14) Liu, Y. M.; Xia, D.; Li, B. W.; Zhang, Q. Y.; Sakurai, T.; Tan, Y. Z.; Seki, S.; Xie, S. Y.; Zheng, L. S., Functional Sulfur-Doped Buckybowls and Their Concave-Convex Supramolecular Assembly with Fullerenes. Angew. Chem. Int. Ed. 2016, 55, 13047-13051.

(15) Josa, D.; Rodríguez-Otero, J.; Cabaleiro-Lago, E. M., Fullerene recognition with molecular tweezers made up of efficient buckybowls: a dispersion-corrected DFT study. Phys. Chem. Chem. Phys. 2015, 17, 13206-13214.

(16) Jackson, E. A.; Steinberg, B. D.; Bancu, M.; Wakamiya, A.; Scott, L. T., Pentaindenocorannulene and Tetraindenocorannulene: New Aromatic Hydrocarbon $\pi$ Systems with Curvatures Surpassing That of C60. J. Am. Chem. Soc. 2007, 129, 484-485.

(17) Wu, T.-C.; Chen, M.-K.; Lee, Y.-W.; Kuo, M.-Y.; Wu, Y.-T., Bowl-Shaped Fragments of C70 or Higher Fullerenes: Synthesis, Structural Analysis, and Inversion Dynamics. Angew. Chem. Int. Ed. 2013, 52, 1289-1293.

(18) Wu, T.-C.; Hsin, H.-J.; Kuo, M.-Y.; Li, C.-H.; Wu, Y.-T., Synthesis and Structural Analysis of a Highly Curved Buckybowl Containing Corannulene and Sumanene Fragments. J. Am. Chem. Soc. 2011, 133, 16319-16321.

(19) Sakurai, H.; Daiko, T.; Hirao, T., A Synthesis of Sumanene, a Fullerene Fragment. Science 2003, 301, 1878-1878.

(20) Whalley, A. C.; Plunkett, K. N.; Gorodetsky, A. A.; Schenck, C. L.; Chiu, C.-Y.; Steigerwald, M. L.; Nuckolls, C., Bending contorted hexabenzocoronene into a bowl. Chem. Sci. 2011, 2, 132-135.

(21) Petrukhina, M. A.; Andreini, K. W.; Peng, L.; Scott, L. T., Hemibuckminsterfullerene C30H12: Xray Crystal Structures of the Parent Hydrocarbon and of the Two-Dimensional Organometallic Network \{[Rh2(O2CCF3)4]3·(C3OH12)\}. Angew. Chem. Int. Ed. 2004, 43, 5477-5481.

(22) Sygula, A.; Saeb $\varnothing$, S., $\pi-\pi$ Stacking of curved carbon networks: The corannulene dimer. Int. J. Quantum Chem. 2009, 109, 65-72.

(23) Josa, D.; González-Veloso, I.; Rodríguez-Otero, J.; Cabaleiro-Lago, E. M., Tailoring buckybowls for fullerene recognition. A dispersion-corrected DFT study. Phys. Chem. Chem. Phys. 2015, 17, 6233-6241.

(24) Josa, D.; Rodríguez-Otero, J.; Cabaleiro-Lago, E. M., A DFT study of substituent effects in corannulene dimers. Phys. Chem. Chem. Phys. 2011, 13, 21139-21145.

(25) Kennedy, M. R.; Burns, L. A.; Sherrill, C. D., Buckyplates and Buckybowls: Examining the Effects of Curvature on $\pi-\pi$ Interactions. J. Phys. Chem. A 2012, 116, 11920-11926. 
(26) Gallego, M.; Calbo, J.; Aragó, J.; Krick Calderon, R. M.; Liquido, F. H.; Iwamoto, T.; Greene, A. K.; Jackson, E. A.; Pérez, E. M.; Ortí, E.; Guldi, D. M.; Scott, L. T.; Martín, N., Electron Transfer in a Supramolecular Associate of a Fullerene Fragment. Angew. Chem. Int. Ed. 2014, 53, 2170-2175.

(27) Calbo, J.; Sancho-García, J. C.; Ortí, E.; Aragó, J., DLPNO-CCSD(T) scaled methods for the accurate treatment of large supramolecular complexes. J. Comput. Chem. 2017, 38, 1869-1878.

(28) Gallego, M.; Calbo, J.; Krick Calderon, R. M.; Pla, P.; Hsieh, Y.-C.; Pérez, E. M.; Wu, Y.-T.; Ortí, E.; Guldi, D. M.; Martín, N., Complexation and Electronic Communication between CorannuleneBased Buckybowls and a Curved Truxene-TTF Donor. Chem. Eur. J. 2017, 23, 3666-3673.

(29) Contreras-García, J.; Johnson, E. R.; Keinan, S.; Chaudret, R.; Piquemal, J.-P.; Beratan, D. N.; Yang, W., NCIPLOT: A Program for Plotting Noncovalent Interaction Regions. J. Chem. Theory Comput. 2011, 7, 625-632.

(30) Johnson, E. R.; Keinan, S.; Mori-Sánchez, P.; Contreras-García, J.; Cohen, A. J.; Yang, W., Revealing Noncovalent Interactions. J. Am. Chem. Soc. 2010, 132, 6498-6506.

(31) Bader, R. F. W., Atoms in Molecules: A Quantum Theory. Clarendon Press: 1994.

(32) Keith, T. A. AIMAll (Version 17.01.25), TK Gristmill Software: Overland Park KS, USA, 2017, (aim.tkgristmill.com).

(33) Contreras-García, J.; Boto, R. A.; Izquierdo-Ruiz, F.; Reva, I.; Woller, T.; Alonso, M., A benchmark for the non-covalent interaction $(\mathrm{NCl})$ index or... is it really all in the geometry? Theor. Chem. Acc. 2016, 135, 242.

(34) Frisch, M. J.; Trucks, G. W.; Schlegel, H. B.; Scuseria, G. E.; Robb, M. A.; Cheeseman, J. R.; Scalmani, G.; Barone, V.; Mennucci, B.; Petersson, G. A.; Nakatsuji, H.; Caricato, M.; Li, X.; Hratchian, H. P.; Izmaylov, A. F.; Bloino, J.; Zheng, G.; Sonnenberg, J. L.; Hada, M.; Ehara, M.; Toyota, K.; Fukuda, R.; Hasegawa, J.; Ishida, M.; Nakajima, T.; Honda, Y.; Kitao, O.; Nakai, H.; Vreven, T.; Montgomery, J. A.; Peralta, J. E.; Ogliaro, F.; Bearpark, M.; Heyd, J. J.; Brothers, E.; Kudin, K. N.; Staroverov, V. N.; Kobayashi, R.; Normand, J.; Raghavachari, K.; Rendell, A.; Burant, J. C.; lyengar, S. S.; Tomasi, J.; Cossi, M.; Rega, N.; Millam, J. M.; Klene, M.; Knox, J. E.; Cross, J. B.; Bakken, V.; Adamo, C.; Jaramillo, J.; Gomperts, R.; Stratmann, R. E.; Yazyev, O.; Austin, A. J.; Cammi, R.; Pomelli, C.; Ochterski, J. W.; Martin, R. L.; Morokuma, K.; Zakrzewski, V. G.; Voth, G. A.; Salvador, P.; Dannenberg, J. J.; Dapprich, S.; Daniels, A. D.; Farkas; Foresman, J. B.; Ortiz, J. V.; Cioslowski, J.; Fox, D. J. Gaussian 09, Revision D.01, Wallingford CT, 2009.

(35) Gonthier, J. F.; Steinmann, S. N.; Roch, L.; Ruggi, A.; Luisier, N.; Severin, K.; Corminboeuf, C., pi-Depletion as a criterion to predict pi-stacking ability. Chem. Commun. 2012, 48, 9239-9241.

(36) Schmider, H. L.; Becke, A. D., Chemical content of the kinetic energy density. Theochem-J. Mol. Struct. 2000, 527, 51-61.

(37) Kohout, M. DGrid, version 4.6, Radebeul, 2011.

(38) Boto, R. A.; Contreras-García, J.; Tierny, J.; Piquemal, J.-P., Interpretation of the reduced density gradient. Mol. Phys. 2016, 114, 1406-1414.

(39) Mitoraj, M. P.; Michalak, A.; Ziegler, T., A Combined Charge and Energy Decomposition Scheme for Bond Analysis. J. Chem. Theory Comput. 2009, 5, 962-975.

(40) Neese, F., The ORCA program system. WIREs Comput. Mol. Sci. 2012, 2, 73-78.

(41) Řezáč, J.; Riley, K. E.; Hobza, P., S66: A Well-balanced Database of Benchmark Interaction Energies Relevant to Biomolecular Structures. J. Chem. Theory Comput. 2011, 7, 2427-2438.

(42) Burns, L. A.; Marshall, M. S.; Sherrill, C. D., Comparing Counterpoise-Corrected, Uncorrected, and Averaged Binding Energies for Benchmarking Noncovalent Interactions. J. Chem. Theory Comput. 2014, 10, 49-57. 
TABLE OF CONTENTS

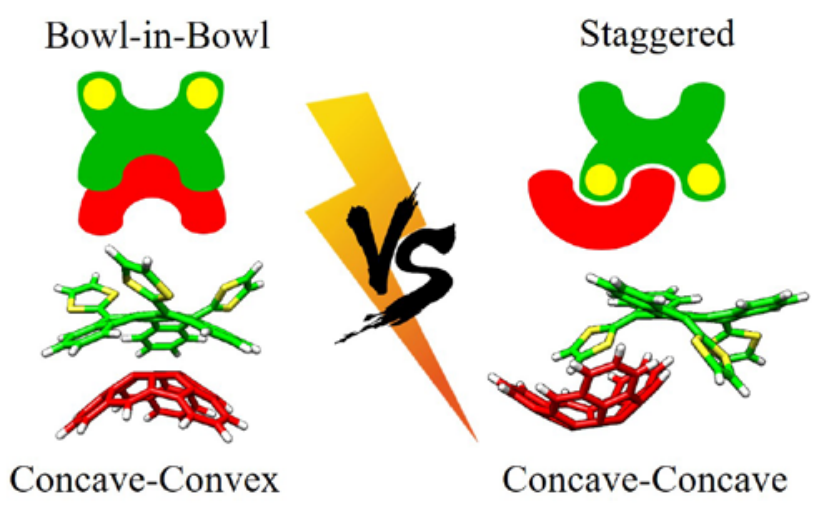

